\title{
Wave diffraction by multiple arbitrary shaped cracks in an infinitely extended ice sheet of finite water depth
}

\author{
Zhi Fu Li ${ }^{1}$, Guo Xiong $\mathrm{Wu}^{2} \dagger$ and Kang Ren ${ }^{2}$ \\ ${ }^{1}$ School of Naval Architecture and Ocean Engineering, Jiangsu University of Science and \\ Technology, Zhenjiang 212003, China \\ ${ }^{2}$ Department of Mechanical Engineering, University College London, Torrington Place, London \\ WC1E $7 \mathrm{JE}, \mathrm{UK}$
}

(Received $\mathrm{xx}$; revised $\mathrm{xx}$; accepted $\mathrm{xx}$ )

Flexural gravity wave interactions with multiple cracks in an ice sheet of infinite extent are considered, based on the linearized velocity potential theory for fluid flow and thin elastic plate model for the ice sheet. Both the shape and location of the cracks can be arbitrary, while an individual crack can be either open or closed. Free edge conditions are imposed at the crack. For open cracks, zero corner force conditions are further applied at the crack tips. The solution procedure starts from series expansion in the vertical direction based on separation of variables, which decomposes the three dimensional problem into an infinite number of coupled two dimensional problems in the horizontal plane. For each two dimensional problem, integral equation is derived along the cracks, with the jumps of displacement and slope of the ice sheet as unknowns in the integrand. By extending the crack in the vertical direction into the fluid domain, an artificial vertical surface is formed, on which an orthogonal inner product is adopted for the vertical modes. Through this, the edge conditions at the cracks are satisfied, together with continuous conditions of pressure and velocity on the vertical surface. The integral differential equations are solved numerically through the boundary element method together with the finite difference scheme for the derivatives along the crack. Extensive results are provided and analyzed for cracks with various shapes and locations, including the jumps of displacement and slope, diffraction wave coefficient, and the scattered cross section.

Key words: Authors should not enter keywords on the manuscript, as these must be chosen by the author during the online submission process and will then be added during the typesetting process (see http://journals.cambridge.org/data/relatedlink/jfmkeywords.pdf for the full list)

\section{Introduction}

A typical feature of both Polar regions is that vast of ocean is covered by ice. Observation showed that the waves generated in open sea could penetrate far into the ice-covered region (Squire et al. 1995). The feature of the wave will change due to its interaction with the ice sheet. One example is that the reduction of the ice extent and thickness will lead to an overall uptrend of the wave height. The topic of coupled ocean

$\dagger$ Email address for correspondence: g.wu@ucl.ac.uk 
wave/sea ice interactions has increasingly attracted research interests over the last two decades. This is due to the fact the Polar ice cover can be greatly affected by the global climate change, which will in turn affect the global environment (Squire 2020). It is also due to the requirement of safe navigation of the potential shipping routes and offshore operations in Arctic region (Eliasson et al. 2017).

Field experiments conducted by Robin (1963) and Squire et al. (1988) have confirmed that the large ice sheet would bend to let the energy pass through in form of flexuralgravity waves. Based on the linear velocity potential theory for fluid flow and thin elastic plate model for ice sheet deflection, a number of solution approaches have been developed for wave scattering in icy waters. When waves propagate from open sea to the ice-covered water or the other way around, the energy will be partially reflected and transmitted due to the change of the dispersion relation. The problem of wave interactions with a homogeneous semi-infinite ice sheet, was solved for the normal incident wave by Fox \& Squire (1990) and for the oblique incident wave by Fox \& Squire (1994), based on the method of matched eigenfunction expansions (MEE). It was found that strong transmission would be more likely for the long wave. For oblique case, when the incident wave angle was larger than a critical value, no wave would transmit into the ice. MEE was also used by Sahoo et al. (2001) to the same problem, but with the unknowns found through introducing an orthogonal inner product. In addition, the problem can be solved by the Wiener-Hopf method (WHM), as was given by an unpublished report of Evans \& Davies (1968). Later, some modifications were introduced into the WHM, which enabled the numerical computations much easier to be carried out, e.g. Balmforth \& Craster (1999), Chung \& Fox (2002), and Tkacheva (2001, 2004). In Balmforth \& Craster (1999), the Timoshenko-Mindlin model, which further included the effects of rotary inertia and transverse shear of the plate, was adopted for the ice sheet. The authors then showed that the Kirchhoff-Love model for thin elastic plate would give similar results to those by Timoshenko-Mindlin model for a wide range of thickness of the ice sheet. In addition to the MEE and WHM, Linton \& Chung (2003) applied the residue calculus technique (RCT) to the semi-infinite ice sheet, and obtained solutions which were equivalent to those provided by WHM. In some cases, the ice sheet may not extend to infinity. It may have a section of free surface and then continue with the ice cover. Chung \& Linton (2005) considered wave propagations through an ice polynya by RCT, and found that perfect transmission could occur at an infinite number of discrete wave frequencies. Based on the Green function method (GFM), Squire \& Dixon (2001b) studied flexural gravity wave interactions with an embedded iceberg. It may be noted that GFM can be also used to solve the wave propagation through an ice floe, as for example in Meylan \& Squire (1994). Williams \& Squire (2006) considered a more general problem by RCT and WHM, i.e. three connected ice sheets with the first and last ones to be semi-infinite. The above individual scatters can be also assembled together to model the wave evolutions through icy waters in a large scale, as has been done in Bennetts \& Squire (2012).

Apart from the above two dimensional (2D) problems, three dimensional (3D) problems have also been considered, which reflect more accurately the physics in many cases. For a circular ice floe, Meylan \& Squire (1996) solved the problem by the boundary integral equation method, based on either the eigenfunction expansions of the potential and its normal derivative on the circular ice floe or writing the normal derivative in terms of another integral equation. The problem was then extended to the finite water depth by Peter et al. (2004). To simulate the propagation of waves through marginal ice zone (MIZ), ice floe with complicated shape needs to be considered. This was done by Meylan (2002) and Wang \& Meylan (2004) through boundary element method for fluid flow and finite element method for ice sheet. Instead of determining the free modes of vibration 
or the dry modes of the floe independently, Bennetts \& Williams (2010) solved the fluid motion and ice sheet flexure together, i.e. the governing equation for ice sheet flexure was incorporated into the water surface condition for fluid flow through kinematic and dynamic conditions at the interface, as done in most of other works. In Bennetts \& Williams (2010), the wave interactions with a polynya embedded in an infinite ice sheet was also solved. Based on the solution of a single ice floe and the slab clustering method (SCM) originally for multiple structures interaction in open sea, Montiel et al. (2016) investigated the wave spectra evolution features in the MIZ which were comprised of tens of thousands ice floes.

Ice sheets in nature are rarely homogeneous and perfect. One of the imperfections is the crack. Based on the MEE originally developed by Fox \& Squire (1990), Barrett \& Squire (1996) solved the problem of an infinitely long straight line crack in ice sheet on the water surface. Numerical results showed that for short waves most of the energy would be reflected back, which was similar to the case of wave impinging a semi-infinite ice sheet. It was found that there would be a frequency at which perfect transmission would occur. Based on the Green function for ice sheet without the crack, analytical solution for reflection and transmission coefficients could be derived, e.g. by Squire \& Dixon (2000) for a single crack through an explicit formulae and by Squire \& Dixon (2001a) for multiple cracks through a matrix equation. Single or multiple parallel infinitely long cracks were also solved by Evans \& Porter (2003) and Porter \& Evans (2006) for finite water depth. Li et al. (2018b) derived a Green function which satisfied the conditions at the crack. This enabled the expression for the diffraction potential to be written explicitly. The advantage of this method was that it allowed multipole for a circle to be constructed directly ( $\mathrm{Li}$ et al. 2018b), and the obtained Green function could be easily extended to multiple cracks and used for a body of arbitrary shape Li et al. (2018a).

For the 3D problems, the work has been mainly limited to the cracks of some special shapes. Porter \& Evans (2007) considered parallel straight line cracks. The jumps of the displacement and slope of the crack deflection were expanded into Chebyshev polynomials, which took into account the behaviour at the crack ends. Li et al. (2018c) considered a circular crack. The jumps of the displacement and slope of the crack deflection were expanded into Fourier series and the unknown coefficients were found explicitly. In addition to parallel straight lines and closed circles, the cracks in Polar regions may have other shapes. Also, when there is a high concentration of ice floes and the gaps between floes are very narrow, this could be treated as a crack problem. For a crack in a general shape, the problem becomes a major challenge. One of the difficulties is that the free edge conditions at the crack contain high derivatives. When this is applied to an already singular boundary integral equation, it becomes a high order hyper singular equation. The solution of such an equation is not easy to obtain and therefore the physics in such a case is not easy to capture. In this work, we shall develop a methodology to overcome the difficulty, and then solve the problem of wave interactions with multiple cracks of arbitrary shapes and locations. An individual crack can be either closed or open. In the methodology, we extend the crack in the vertical direction into the fluid domain to form an artificial vertical surface. On this surface, continuity of the velocity and pressure of the fluid will be imposed. The 3D problem is then decomposed into an infinite number of coupled $2 \mathrm{D}$ problems. The methodology is verified through the analytical solution for the circular crack and then used for a variety of case studies.

The paper is organized as follows. The mathematical problem for flexural gravity wave propagations in ice sheet with multiple arbitrary shaped cracks is formulated in $\S 2$, and the conditions satisfied at the crack together with those at the ends of an open crack are described. The solution procedures are constructed in $\S 3$, and the numerical discretization 


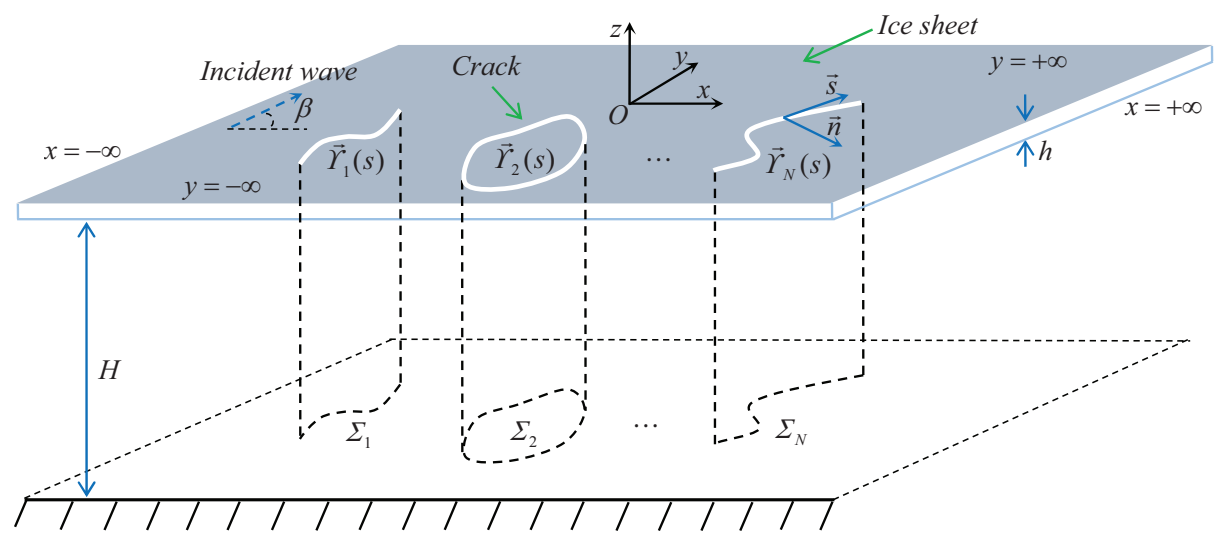

FiguRE 1. Coordinate system and sketch of the problem.

of the hypersingular integral differential equations are given in $\S 4$. Extensive results for cracks with various shapes and distributions are presented and discussed in $\S 5$, and conclusions are made in $\S 6$. In Appendix, the diffraction coefficient corresponding to the far field diffracted wave is derived, and an energy conservation relationship for the scattered cross section is provided.

\section{Mathematical model}

We consider the problem of wave interaction with multiple cracks of arbitrary shapes in an ice sheet, as sketched in figure 1. The ice sheet is horizontally infinitely extended, and floating on water of finite depth. To describe the problem, a Cartesian coordinate system $O-x y z$ is defined, with $O-x y$ plane being the undisturbed interface of water and ice sheet, and $z$-axis pointing vertically upwards. The direction of flexural-gravity incident wave from infinity is assumed to form an angle $\beta$ with positive $x$-axis, and the water depth $H$ is assumed to be constant, as shown in the figure. The shape of crack $i$ can be described parametrically by

$$
\vec{\Upsilon}_{i}(s)=(x(s), y(s)), \quad\left(-\gamma_{i}<s<+\gamma_{i}, 1 \leqslant i \leqslant N\right),
$$

where $2 \gamma_{i}$ is the total arc length of crack $i$, and $s$ is the curvilinear coordinate along the crack.

Based on the assumption that the fluid is inviscid, incompressible and homogeneous, and its motion is irrotational, the velocity potential $\Phi$ can be introduced to describe the fluid flow. When the amplitude of wave motion is small compared to its length, the linearized velocity potential theory can be further used. For sinusoidal motion in time with radian frequency $\omega$, we may write the total velocity potential as

$$
\Phi(x, y, z, t)=\operatorname{Re}\left[\phi(x, y, z) \mathrm{e}^{\mathrm{i} \omega t}\right]
$$

where

$$
\phi=\phi_{I}+\phi_{D}
$$

with $\phi_{I}$ and $\phi_{D}$ as the incident velocity potential and diffracted velocity potential by the cracks respectively. The conservation of mass requires that the velocity potential $\phi$ should satisfy the following Laplace equation

$$
\nabla^{2} \phi+\frac{\partial^{2} \phi}{\partial z^{2}}=0
$$


throughout the fluid, where

$$
\nabla^{2}=\frac{\partial^{2}}{\partial x^{2}}+\frac{\partial^{2}}{\partial y^{2}}
$$

is the Laplacian in the horizontal plane. Following Squire (2011) and others, the ice sheet is modelled as a thin elastic plate, and its properties (i.e. Young's modulus E, Poisson's ratio $\nu$, density $\rho_{i}$ and thickness $h$ ) are assumed to be constant. Assuming that there is no gap between the ice sheet and the water upper surface, we can write the boundary condition on their interface as

$$
\left(L \nabla^{4}+\rho_{w} g-m \omega^{2}\right) \frac{\partial \phi}{\partial z}-\rho_{w} \omega^{2} \phi=0, \quad\left(\vec{r} \neq \vec{\Upsilon}_{i}, z=0\right),
$$

where $\vec{r}=(x, y), L=E h^{3} /\left[12\left(1-\nu^{2}\right)\right]$ is the effective flexural rigidity of the ice sheet, $m=\rho_{i} h$ is the corresponding areal density, $\rho_{w}$ is the density of water, and $g$ is the acceleration due to gravity. On both sides of the crack, the edges of the ice sheet are assumed to be free to move, which means that the following zero bending moment and modified shear force conditions should be satisfied (Timoshenko \& Woinowsky 1959)

$$
\mathcal{B}\left(\frac{\partial \phi}{\partial z}\right)=0 \quad \text { and } \quad \mathcal{S}\left(\frac{\partial \phi}{\partial z}\right)=0, \quad\left(\vec{r}=\vec{\Upsilon}_{i}, z=0\right)
$$

where the operator $\mathcal{B}$ and $\mathcal{S}$ are respectively defined as

$$
\begin{gathered}
\mathcal{B}=\nabla^{2}-\nu_{0}\left(\sin ^{2} \Theta \frac{\partial^{2}}{\partial x^{2}}+\cos ^{2} \Theta \frac{\partial^{2}}{\partial y^{2}}-\sin 2 \Theta \frac{\partial^{2}}{\partial x \partial y}\right), \\
\mathcal{S}=\frac{\partial}{\partial n} \nabla^{2}+\nu_{0} \frac{\partial}{\partial s}\left[\cos 2 \Theta \frac{\partial^{2}}{\partial x \partial y}+\frac{\sin 2 \Theta}{2}\left(\frac{\partial^{2}}{\partial y^{2}}-\frac{\partial^{2}}{\partial x^{2}}\right)\right],
\end{gathered}
$$

with $\nu_{0}=1-\nu$, and $\vec{n}$ and $\vec{s}$ are the unit vectors along the normal and tangential directions, respectively. Here, $\Theta(s)$ is the angle between positive $\vec{n}$ and $x$-axis, and thus $\vec{n}=(\cos \Theta, \sin \Theta)$ and $\vec{s}=(-\sin \Theta, \cos \Theta)$. By noticing

$$
\frac{\partial x}{\partial s}=-\sin \Theta \quad \text { and } \quad \frac{\partial y}{\partial s}=\cos \Theta
$$

the operators $\mathcal{B}$ and $\mathcal{S}$ in (2.8) and (2.9) can be also expressed into the curvilinear coordinate system as

$$
\begin{gathered}
\mathcal{B}=\nabla^{2}-\nu_{0}\left(\frac{\partial^{2}}{\partial s^{2}}+\frac{\partial \Theta}{\partial s} \frac{\partial}{\partial n}\right), \\
\mathcal{S}=\frac{\partial}{\partial n} \nabla^{2}+\nu_{0} \frac{\partial}{\partial s}\left(\frac{\partial^{2}}{\partial s \partial n}-\frac{\partial \Theta}{\partial s} \frac{\partial}{\partial s}\right),
\end{gathered}
$$

where the partial derivatives with respect to $s$ are carried out with respect to the curvilinear coordinate. It should be noticed that equation (2.12) is in fact an equivalent shear force after three boundary conditions at a plate edge being combined into two. The second term in (2.12) is from the twisting moment, which is equivalently replaced by the force perpendicular to the plate. In doing so, when a plate edge has a sharp corner, it leads to a concentrated force at the corner, which has to be set as a zero as an additional condition. For an open crack, at the ends of the crack the free edge in fact turns $360^{\circ}$. Thus this additional condition should be imposed at the sharp corner of a plate (Timoshenko \& Woinowsky 1959)

$$
\left(\frac{\partial^{2}}{\partial s \partial n}-\frac{\partial \Theta}{\partial s} \frac{\partial}{\partial s}\right) \frac{\partial \phi}{\partial z}=0, \quad\left(s \rightarrow \pm \gamma_{i}, z=0\right) .
$$


The edge conditions described above are on the basis that the ice sheets on both sides of the crack are completely detached from each other, which is suitable when the ratio of width of the gap to the typical length scale of the ice sheet is small ( $\mathrm{Li}$ et al. 2018b). It might be noticed that the implications of the mathematical model for the ice crack have been reviewed and discussed by Squire (2007). On the flat seabed, the impermeable condition provides

$$
\frac{\partial \phi}{\partial z}=0, \quad(z=-H)
$$

At infinity the radiation condition is imposed, which requires that the diffracted wave should propagate outwards, or

$$
\lim _{r \rightarrow \infty} \sqrt{r}\left(\frac{\partial \phi_{D}}{\partial r}+\mathrm{i} \kappa_{0} \phi_{D}\right)=0
$$

where $\kappa_{0}$ is the flexural gravity wave number. It may be noticed that $\kappa_{0}$ is the purely positive real root of the dispersion equation $K(\omega, \kappa)=0$ for flexural gravity wave in the ice sheet, with

$$
K(\omega, \kappa) \equiv\left(L \kappa^{4}+\rho_{w} g-m \omega^{2}\right) \kappa \tanh (\kappa H)-\rho_{w} \omega^{2}
$$

\section{Solution procedures}

To solve the boundary value problem described in the section above, through using variable separation method, we may write the diffracted velocity potential $\phi_{D}$ into the following form

$$
\phi_{D}=\sum_{m} \varphi_{m}(x, y) \psi_{m}(z)
$$

Substituting (3.1) into the governing Laplace equation (2.4), we can obtain $\left(\nabla^{2} \varphi_{m}\right) / \varphi_{m}=$ $-\left(\mathrm{d}^{2} \psi_{m} / \mathrm{d} z^{2}\right) / \psi_{m}$. This equation has to be equal to a constant. Letting it be $-\kappa_{m}^{2}$, we have

$$
\nabla^{2} \varphi_{m}+\kappa_{m}^{2} \varphi_{m}=0
$$

and

$$
\frac{\mathrm{d}^{2} \psi_{m}}{\mathrm{~d} z^{2}}-\kappa_{m}^{2} \psi_{m}=0
$$

for $\varphi_{m}$ and $\psi_{m}$ respectively. By further applying the boundary conditions in (2.6) and (2.14) to (3.3), we have

$$
\psi_{m}(z)=\frac{\cosh \left[\kappa_{m}(z+H)\right]}{\cosh \left(\kappa_{m} H\right)} .
$$

Here, $\kappa_{m}$ are the roots of the dispersion equation (2.16), with $\kappa_{-2}$ and $\kappa_{-1}$ as two complex roots with negative imaginary parts and symmetric about the imaginary axis, $\kappa_{0}$ as the purely positive real root, $\kappa_{m}(m=1, \ldots, \infty)$ as an infinite number of purely negative imaginary roots. Taking the summation in (3.1) through all possible eigen values and eigen functions, we have

$$
\phi_{D}=\sum_{m=-2}^{\infty} \varphi_{m}(x, y) \psi_{m}(z) .
$$

Then the three dimensional boundary value problems for $\phi_{D}$ are transformed into the two dimensional ones for $\varphi_{m}$. The incident velocity potential $\phi_{I}$ in (2.3) may be written as

$$
\phi_{I}=\varphi_{I}(x, y) \psi_{0}(z)
$$


where

$$
\varphi_{I}(x, y)=I \mathrm{e}^{-\mathrm{i} \kappa_{0}(x \cos \beta+y \sin \beta)} / \tanh \left(\kappa_{0} H\right),
$$

with $I=\mathrm{i} \omega \Lambda / \kappa_{0}$ and $\Lambda$ as the amplitude of the incident wave.

The two dimensional Helmholtz equation in (3.2) may be converted into an integral equation through using the Green function below

$$
G_{m}(p, q)=\frac{\pi}{2 \mathrm{i}} H_{0}^{(2)}\left(\kappa_{m} R\right),
$$

where $H_{0}^{(2)}$ is the zeroth order Hankel function of second kind (Abramowitz \& Stegun $1965)$, and $R$ is the distance between the field point $p(x, y)$ and source point $q(\xi, \eta)$ in the two dimensional horizontal plane. We may note that $G_{m}$ in (3.8) satisfies the Helmholtz equation and the radiation condition in (2.15). Applying Green's identity to $G_{m}$ and $\varphi_{m}$, we have

$$
\alpha \varphi_{m}(p)=\int_{\Gamma}\left[G_{m}(p, q) \frac{\partial \varphi_{m}(q)}{\partial n_{q}}-\frac{\partial G_{m}(p, q)}{\partial n_{q}} \varphi_{m}(q)\right] \mathrm{d} s_{q},
$$

where the integral and normal derivative are carried out with respect to the source point $q$, and $\alpha$ is the two dimensional solid angle at the field point $p$. In (3.9), $\Gamma$ should include all the lines along the ice sheet edges. However, the closed line at infinity has been excluded due to the fact that both $G_{m}$ and $\varphi_{m}$ satisfy the radiation condition in (2.15), and the integrand becomes zero there. For ice crack, we may denote the variable on its two sides with superscripts + and - respectively. Noticing that on + and - side $\vec{n}$ in (3.9) is opposite to each other, it can be rewritten as

$$
\alpha \varphi_{m}(p)=\sum_{i=1}^{N} \int_{\Gamma_{i}}\left[G_{m}(p, q) \frac{\partial \tilde{\varphi}_{m}(q)}{\partial n_{q}}-\frac{\partial G_{m}(p, q)}{\partial n_{q}} \tilde{\varphi}_{m}(q)\right] \mathrm{d} s_{q},
$$

where $\Gamma_{i}$ is the route along the crack $i, \vec{n}$ is now from - side to + side. $\tilde{\varphi}_{m}$ and $\partial \tilde{\varphi}_{m} / \partial n$ are the corresponding jumps of velocity potential and its normal derivative across the crack, or

$$
\begin{gathered}
\tilde{\varphi}_{m}=\varphi_{m}^{-}-\varphi_{m}^{+}, \\
\frac{\partial \tilde{\varphi}_{m}}{\partial n}=\frac{\partial \varphi_{m}^{-}}{\partial n}-\frac{\partial \varphi_{m}^{+}}{\partial n} .
\end{gathered}
$$

Once the values of $\tilde{\varphi}_{m}$ and $\partial \tilde{\varphi}_{m} / \partial n$ along the cracks are determined, the diffraction potential $\phi_{D}$ in any point of fluid can be obtained through equations (3.5) and (3.10). The main task is then to find these two jumps. To do that, we extend each ice crack vertically from the ice sheet to the sea bed to form a vertical surface $\Sigma_{i}$, as shown in figure 1 . On both sides of the artificial surface $\Sigma_{i}$, we denote the total velocity potential $\phi$ as $\phi^{+}$and $\phi^{-}$respectively, where the superscripts correspond to those of $\varphi_{m}$. In fluid, the pressure and fluid velocity should be continuous across each $\Sigma_{i}$, or

$$
\begin{gathered}
\phi^{+}=\phi^{-}, \\
\frac{\partial \phi^{+}}{\partial n}=\frac{\partial \phi^{-}}{\partial n},
\end{gathered}
$$

for $-H \leqslant z \leqslant 0$. Different from the free surface problem, the eigenfunction in (3.4) is not orthogonal in the usual sense. However, we may introduce the following orthogonal inner product (Sahoo et al. 2001)

$$
\left\langle\psi_{m}, \psi_{\tilde{m}}\right\rangle=\int_{-H}^{0} \psi_{m} \psi_{\tilde{m}} \mathrm{~d} z+\frac{L}{\rho_{w} \omega^{2}}\left(\frac{\mathrm{d} \psi_{m}}{\mathrm{~d} z} \frac{\mathrm{d}^{3} \psi_{\tilde{m}}}{\mathrm{~d} z^{3}}+\frac{\mathrm{d}^{3} \psi_{m}}{\mathrm{~d} z^{3}} \frac{\mathrm{d} \psi_{\tilde{m}}}{\mathrm{~d} z}\right)_{z=0},
$$


which provides that $\left\langle\psi_{m}, \psi_{\tilde{m}}\right\rangle=0$ if $m \neq \tilde{m}$ and $\left\langle\psi_{m}, \psi_{\tilde{m}}\right\rangle=Q_{m}$ if $m=\tilde{m}$, with

$$
Q_{m}=\frac{2 \kappa_{m} H+\sinh \left(2 \kappa_{m} H\right)}{4 \kappa_{m} \cosh ^{2}\left(\kappa_{m} H\right)}+\frac{2 L \kappa_{m}^{4}}{\rho_{w} \omega^{2}} \tanh ^{2}\left(\kappa_{m} H\right) .
$$

To satisfy the continuity condition of pressure in (3.13), applying the inner product to $\phi^{-}-\phi^{+}$and $\psi_{\tilde{m}}$, we have

$$
\begin{aligned}
\left\langle\left(\phi^{-}-\phi^{+}\right), \psi_{\tilde{m}}\right\rangle= & \int_{-H}^{0}\left(\phi^{-}-\phi^{+}\right) \psi_{\tilde{m}} \mathrm{~d} z \\
& +\frac{L}{\rho_{w} \omega^{2}}\left[\frac{\partial\left(\phi^{-}-\phi^{+}\right)}{\partial z} \frac{\mathrm{d}^{3} \psi_{\tilde{m}}}{\mathrm{~d} z^{3}}+\frac{\partial^{3}\left(\phi^{-}-\phi^{+}\right)}{\partial z^{3}} \frac{\mathrm{d} \psi_{\tilde{m}}}{\mathrm{~d} z}\right]_{z=0} .
\end{aligned}
$$

Similarly, for the continuity condition of velocity in (3.14), we have

$$
\begin{aligned}
\left\langle\frac{\partial\left(\phi^{-}-\phi^{+}\right)}{\partial n}, \psi_{\tilde{m}}\right\rangle= & \int_{-H}^{0} \frac{\partial\left(\phi^{-}-\phi^{+}\right)}{\partial n} \psi_{\tilde{m}} \mathrm{~d} z \\
& +\frac{L}{\rho_{w} \omega^{2}}\left[\frac{\partial^{2}\left(\phi^{-}-\phi^{+}\right)}{\partial z \partial n} \frac{\mathrm{d}^{3} \psi_{\tilde{m}}}{\mathrm{~d} z^{3}}+\frac{\partial^{4}\left(\phi^{-}-\phi^{+}\right)}{\partial z^{3} \partial n} \frac{\mathrm{d} \psi_{\tilde{m}}}{\mathrm{~d} z}\right]_{z=0}
\end{aligned}
$$

In the above two equations, $z=0$ should be understood as that the crack is approached from ice sheet not from $\Sigma_{i}$, and therefore jumps exist. Substituting equations (3.5) and (3.6) into (2.3), and further the obtained results into (3.17) and (3.18), we can get

$$
\tilde{\varphi}_{\tilde{m}} Q_{\tilde{m}}=\frac{L}{\rho_{w} \omega^{2}}\left[\tilde{D} \frac{\mathrm{d}^{3} \psi_{\tilde{m}}}{\mathrm{~d} z^{3}}+\frac{\partial^{3}\left(\phi_{D}^{-}-\phi_{D}^{+}\right)}{\partial z^{3}} \frac{\mathrm{d} \psi_{\tilde{m}}}{\mathrm{~d} z}\right]_{z=0}
$$

and

$$
\frac{\partial \tilde{\varphi}_{\tilde{m}}}{\partial n} Q_{\tilde{m}}=\frac{L}{\rho_{w} \omega^{2}}\left[\tilde{S} \frac{\mathrm{d}^{3} \psi_{\tilde{m}}}{\mathrm{~d} z^{3}}+\frac{\partial^{4}\left(\phi_{D}^{-}-\phi_{D}^{+}\right)}{\partial z^{3} \partial n} \frac{\mathrm{d} \psi_{\tilde{m}}}{\mathrm{~d} z}\right]_{z=0},
$$

where the continuity conditions (3.13) and (3.14) have been used in the first terms on the right hand sides of $(3.17)$ and (3.18), and $\tilde{D}$ and $\tilde{S}$ are respectively the jumps of displacement and slope across the crack or

$$
\begin{gathered}
\tilde{D}=\left(\frac{\partial \phi_{D}^{-}}{\partial z}-\frac{\partial \phi_{D}^{+}}{\partial z}\right)_{z=0}, \\
\tilde{S}=\left(\frac{\partial^{2} \phi_{D}^{-}}{\partial n \partial z}-\frac{\partial^{2} \phi_{D}^{+}}{\partial n \partial z}\right)_{z=0} .
\end{gathered}
$$

It may be noticed that in equations (3.19) and (3.20), the continuity of $\varphi_{I}$ in (3.6) across the artificial vertical surface $\Sigma_{i}$ has been implied. Since $\phi_{D}$ should satisfy the Laplace equation in (2.4) or $\partial^{2} \phi_{D} / \partial z^{2}=-\nabla^{2} \phi_{D}$, we may further write (3.19) and (3.20) as

$$
\tilde{\varphi}_{\tilde{m}} Q_{\tilde{m}}=\frac{L}{\rho_{w} \omega^{2}}\left[\tilde{D} \frac{\mathrm{d}^{3} \psi_{\tilde{m}}}{\mathrm{~d} z^{3}}-\frac{\mathrm{d} \psi_{\tilde{m}}}{\mathrm{~d} z} \nabla^{2} \frac{\partial\left(\phi_{D}^{-}-\phi_{D}^{+}\right)}{\partial z}\right]_{z=0},
$$

and

$$
\frac{\partial \tilde{\varphi}_{\tilde{m}}}{\partial n} Q_{\tilde{m}}=\frac{L}{\rho_{w} \omega^{2}}\left\{\tilde{S} \frac{\mathrm{d}^{3} \psi_{\tilde{m}}}{\mathrm{~d} z^{3}}-\frac{\mathrm{d} \psi_{\tilde{m}}}{\mathrm{~d} z} \frac{\partial}{\partial n}\left[\nabla^{2} \frac{\partial\left(\phi_{D}^{-}-\phi_{D}^{+}\right)}{\partial z}\right]\right\}_{z=0} .
$$

Since both $\phi^{-}$and $\phi^{+}$satisfy the ice sheet edge conditions in (2.7), their difference should 
also satisfy the same edge conditions. By further noticing that

$$
\mathcal{B}\left(\frac{\partial \phi_{I}^{-}}{\partial z}\right)=\mathcal{B}\left(\frac{\partial \phi_{I}^{+}}{\partial z}\right) \quad \text { and } \quad \mathcal{S}\left(\frac{\partial \phi_{I}^{-}}{\partial z}\right)=\mathcal{S}\left(\frac{\partial \phi_{I}^{+}}{\partial z}\right),
$$

we can obtain

$$
\begin{gathered}
\nabla^{2} \frac{\partial\left(\phi_{D}^{-}-\phi_{D}^{+}\right)}{\partial z}=\nu_{0}\left(\frac{\partial^{2} \tilde{D}}{\partial s^{2}}+\frac{\partial \Theta}{\partial s} \tilde{S}\right) \\
\frac{\partial}{\partial n}\left[\nabla^{2} \frac{\partial\left(\phi_{D}^{-}-\phi_{D}^{+}\right)}{\partial z}\right]=-\nu_{0} \frac{\partial}{\partial s}\left(\frac{\partial \tilde{S}}{\partial s}-\frac{\partial \Theta}{\partial s} \frac{\partial \tilde{D}}{\partial s}\right) .
\end{gathered}
$$

Substituting equations (3.26) and (3.27) into (3.23) and (3.24), we have

$$
\tilde{\varphi}_{\tilde{m}}=\frac{L \kappa_{\tilde{m}} \tanh \left(\kappa_{\tilde{m}} H\right)}{Q_{\tilde{m}} \rho_{w} \omega^{2}}\left[\kappa_{\tilde{m}}^{2} \tilde{D}-\nu_{0}\left(\frac{\partial^{2} \tilde{D}}{\partial s^{2}}+\frac{\partial \Theta}{\partial s} \tilde{S}\right)\right],
$$

and

$$
\frac{\partial \tilde{\varphi}_{\tilde{m}}}{\partial n}=\frac{L \kappa_{\tilde{m}} \tanh \left(\kappa_{\tilde{m}} H\right)}{Q_{\tilde{m}} \rho_{w} \omega^{2}}\left[\kappa_{\tilde{m}}^{2} \tilde{S}+\nu_{0} \frac{\partial}{\partial s}\left(\frac{\partial \tilde{S}}{\partial s}-\frac{\partial \Theta}{\partial s} \frac{\partial \tilde{D}}{\partial s}\right)\right] .
$$

Substituting equations (3.28) and (3.29) into (3.10), we can obtain

$$
\begin{aligned}
\varphi_{m}= & \chi_{m} \sum_{i=1}^{N} \int_{\Gamma_{i}}\left\{G_{m}\left[\kappa_{m}^{2} \tilde{S}+\nu_{0} \frac{\partial}{\partial s}\left(\frac{\partial \tilde{S}}{\partial s}-\frac{\partial \Theta}{\partial s} \frac{\partial \tilde{D}}{\partial s}\right)\right]\right. \\
& \left.-\frac{\partial G_{m}}{\partial n_{q}}\left[\kappa_{m}^{2} \tilde{D}-\nu_{0}\left(\frac{\partial^{2} \tilde{D}}{\partial s^{2}}+\frac{\partial \Theta}{\partial s} \tilde{S}\right)\right]\right\} \mathrm{d} s_{q},
\end{aligned}
$$

where

$$
\chi_{m}=\frac{L \kappa_{m} \tanh \left(\kappa_{m} H\right)}{\alpha Q_{m} \rho_{w} \omega^{2}} .
$$

We may apply integration by parts to the second term on the right hand side of $(3.30)$ and use (2.13), which provides

$$
\varphi_{m}=\chi_{m} \sum_{i=1}^{N} \int_{\Gamma_{i}}\left(f_{m}^{D} \tilde{D}+f_{m}^{S} \tilde{S}\right) \mathrm{d} s_{q}
$$

where

$$
\begin{gathered}
f_{m}^{D}=\frac{\partial G_{m}}{\partial s_{q}} \nu_{0} \frac{\partial \Theta}{\partial s} \frac{\partial}{\partial s}-\frac{\partial G_{m}}{\partial n_{q}}\left(\kappa_{m}^{2}-\nu_{0} \frac{\partial^{2}}{\partial s^{2}}\right), \\
f_{m}^{S}=G_{m} \kappa_{m}^{2}+\frac{\partial G_{m}}{\partial n_{q}} \nu_{0} \frac{\partial \Theta}{\partial s}-\frac{\partial G_{m}}{\partial s_{q}} \nu_{0} \frac{\partial}{\partial s} .
\end{gathered}
$$

Taking normal derivation in (3.32) at point $p$, we further have

$$
\frac{\partial \varphi_{m}}{\partial n}=\chi_{m} \sum_{i=1}^{N} \frac{\partial}{\partial n_{p}} \int_{\Gamma_{i}}\left(f_{m}^{D} \tilde{D}+f_{m}^{S} \tilde{S}\right) \mathrm{d} s_{q} .
$$

Now, the integral equations contain the unknown jumps of displacement and slope across the crack. To solve $\tilde{D}$ and $\tilde{S}$, we may substitute equations (3.32) into (3.5) directly to obtain $\phi_{D}$ and (3.35) into the normal derivative of (3.5) to obtain $\partial \phi_{D} / \partial n$, and then 
apply the crack conditions in (2.7), which gives two sets of differential equations, or

$$
\begin{gathered}
\sum_{m=-2}^{\infty}\left[\left(\frac{\mathrm{d}^{3} \psi_{m}}{\mathrm{~d} z^{3}}+\nu_{0} \frac{\mathrm{d} \psi_{m}}{\mathrm{~d} z} \frac{\partial^{2}}{\partial s^{2}}\right) \varphi_{m}+\nu_{0} \frac{\mathrm{d} \psi_{m}}{\mathrm{~d} z} \frac{\partial \Theta}{\partial s} \frac{\partial \varphi_{m}}{\partial n}\right]=\mathcal{B}\left(\frac{\partial \phi_{I}}{\partial z}\right), \\
\sum_{m=-2}^{\infty}\left[\nu_{0} \frac{\mathrm{d} \psi_{m}}{\mathrm{~d} z}\left(\frac{\partial^{2} \Theta}{\partial s^{2}} \frac{\partial}{\partial s}+\frac{\partial \Theta}{\partial s} \frac{\partial^{2}}{\partial s^{2}}\right) \varphi_{m}+\left(\frac{\mathrm{d}^{3} \psi_{m}}{\mathrm{~d} z^{3}}-\nu_{0} \frac{\mathrm{d} \psi_{m}}{\mathrm{~d} z} \frac{\partial^{2}}{\partial s^{2}}\right) \frac{\partial \varphi_{m}}{\partial n}\right]=\mathcal{S}\left(\frac{\partial \phi_{I}}{\partial z}\right),
\end{gathered}
$$

for $\vec{r} \rightarrow \vec{\Upsilon}_{i}$ and $z=0$.

\section{Numerical discretization}

To find the solution for the boundary integral equations (3.32) and (3.35) as well as the differential equations (3.36) and (3.37) numerically, we may divide the $i$-th ice crack into $M_{i}$ straight line segments with length respectively as $\ell_{i}^{1}, \ell_{i}^{2}, \ldots, \ell_{i}^{M_{i}}$. On each segment, the jumps of displacement and slope or $\tilde{D}$ and $\tilde{S}$ are also assumed to be constant and are taken from those at the centre of the segment, where the crack conditions will be enforced. Here, it may be noticed that the solid angle $\alpha$ in the integral equation (3.10) should always be $2 \pi$, even on the crack, due to that the computational domain exists on both sides of the crack, which is different from that for wave interactions with a body of non-zero thickness. For the first and second partial derivatives with respect to $s$, the three point finite difference scheme for unequal segment will be used (Burden \& Faires 2010), or at the $i$-th segment centre with $s=s_{1}$

$$
\left.\frac{\partial f_{i}}{\partial s}\right|_{s=s_{1}}=\frac{-h_{2}^{2} f_{0}-\left(h_{1}-h_{2}\right)\left(h_{1}+h_{2}\right) f_{1}+h_{1}^{2} f_{2}}{h_{1} h_{2}\left(h_{1}+h_{2}\right)},
$$

and

$$
\frac{\partial^{2} f_{i}}{\partial s^{2}}=2 \frac{h_{2} f_{0}-\left(h_{1}+h_{2}\right) f_{1}+h_{1} f_{2}}{h_{1} h_{2}\left(h_{1}+h_{2}\right)},
$$

where $h_{1}=s_{1}-s_{0}$ and $h_{2}=s_{2}-s_{1}, s_{0}$ and $s_{2}$ are the values of $s$ at the previous and next segments respectively. In (4.1) and (4.2), the subscript in $f_{i}$ indicates the values at the $i$-th segment of the crack. Near the edge of an open crack, if there is no required point beyond edge, the one sided 3-point equation can be used, or

$$
\begin{aligned}
& \left.\frac{\partial f_{i}}{\partial s}\right|_{s=s_{0}}=\frac{-h_{2}\left(2 h_{1}+h_{2}\right) f_{0}+\left(h_{1}+h_{2}\right)^{2} f_{1}-h_{1}^{2} f_{2}}{h_{1} h_{2}\left(h_{1}+h_{2}\right)}, \\
& \left.\frac{\partial f_{i}}{\partial s}\right|_{s=s_{2}}=\frac{h_{2}^{2} f_{0}-\left(h_{1}+h_{2}\right)^{2} f_{1}+h_{1}\left(h_{1}+2 h_{2}\right) f_{2}}{h_{1} h_{2}\left(h_{1}+h_{2}\right)} .
\end{aligned}
$$

It should be noticed that in equation (3.35), the normal derivative with respect to $\vec{n}_{p}$ can be moved into the integral. As a result, there is a hypersingular integral which should be computed in the sense of the Hadamard finite part (Martin \& Rizzo 1989). Substituting (3.32) and (3.35) into (3.36) and (3.37), and using (4.1) and (4.2) for the first and second partial derivatives with respect to $s$, we can obtain two sets of linear equations, the total number of which is the same as that for $\tilde{D}$ and $\tilde{S}$.

When the field point $p$ tends to the source point $q$, there will be a logarithmic singularity in the Green function $G_{m}$, as (Abramowitz \& Stegun 1965)

$$
H_{0}^{(2)}\left(\kappa_{m} R\right) \rightarrow \frac{2}{\pi \mathrm{i}} \ln (R)+O(1) \quad \text { as } \quad R \rightarrow 0
$$


In such a case, the integrals in (3.32) and (3.35) should be computed in the principle value or Hadamard finite part sense. For the logarithmic singularity, we may separate it out from $G_{m}$ and integrate it analytically (Linton \& Mciver 2001). To compute the Hadamard finite part integral, we may denote $\vec{n}_{p}=\left(n_{1}^{p}, n_{2}^{p}\right)$ and $\vec{n}_{q}=\left(n_{1}^{q}, n_{2}^{q}\right)$. Then we have

$$
\begin{aligned}
\frac{\partial^{2} G_{m}}{\partial n_{p} \partial n_{q}}= & \frac{\pi \kappa_{m}}{2 \mathrm{i} R} H_{1}^{(2)}\left(\kappa_{m} R\right)\left(\vec{n}_{p} \cdot \vec{n}_{q}\right)+\frac{\pi \kappa_{m}}{2 \mathrm{i} R^{2}}\left[\kappa_{m} H_{0}^{(2)}\left(\kappa_{m} R\right)-\frac{2}{R} H_{1}^{(2)}\left(\kappa_{m} R\right)\right] \\
& \times\left[(\xi-x) n_{1}^{p}+(\eta-y) n_{2}^{p}\right]\left[(\xi-x) n_{1}^{q}+(\eta-y) n_{2}^{q}\right] .
\end{aligned}
$$

For $p$ and $q$ on the same segment, by noticing $\vec{n}_{p}=\vec{n}_{q}$ and $(\xi-x) n_{1}^{p}+(\eta-y) n_{2}^{p}=0$, we may further simplify the above equation as

$$
\frac{\partial^{2} G_{m}}{\partial n_{p} \partial n_{q}}=\frac{\pi \kappa_{m}}{2 \mathrm{i} R} H_{1}^{(2)}\left(\kappa_{m} R\right) .
$$

We may also introduce a local coordinate system $t \in[-1,+1]$. On each panel of crack $i$, we have $R=\ell_{i}|t| / 2$. Thus

$$
\frac{\partial^{2} G_{m}}{\partial n_{p} \partial n_{q}}=\frac{\pi \kappa_{m} H_{1}^{(2)}\left(\kappa_{m} \ell_{i}|t| / 2\right)}{\mathrm{i} \ell_{i}|t|} .
$$

Similar to (4.5), we have for $H_{1}^{(2)}\left(\kappa_{m} R\right)$ (Abramowitz \& Stegun 1965)

$$
H_{1}^{(2)}\left(\kappa_{m} R\right) \rightarrow \frac{\kappa_{m} R}{\pi \mathrm{i}}\left[\ln (R)-\frac{2}{\left(\kappa_{m} R\right)^{2}}\right]+O(R) \quad \text { as } \quad R \rightarrow 0 .
$$

Substituting (4.9) into (4.8), we have

$$
\frac{\partial^{2} G_{m}}{\partial n_{p} \partial n_{q}}=\frac{4}{\ell_{i}^{2} t^{2}}-\frac{\kappa_{m}^{2}}{2} \ln |t|+O(1),
$$

for $R \rightarrow 0$ or $t \rightarrow 0$. Thus, when $p$ and $q$ are located at the same segment, we may divide the integral of (4.8) into the singular part in (4.10) and the remaining regular part with order $O(1)$. The latter one can be computed numerically through the Gauss-Legendre approach. For the former one or the singular part in (4.10), we have

$$
\int_{\ell_{i}} \frac{\partial^{2} G_{m}}{\partial n_{p} \partial n_{q}} \mathrm{~d} s_{q}=\frac{\ell_{i}}{2} \int_{-1}^{+1} \frac{\partial^{2} G_{m}}{\partial n_{p} \partial n_{q}} \mathrm{~d} t=\frac{\kappa_{m}^{2} \ell_{i}}{2}-\frac{4}{\ell_{i}},
$$

where $p$ being located at the centre of $\ell_{i}$ has been assumed. It might be noticed that the singular term in equation (4.11) of order 2 is treated through the Hadamard finite part integral (Martin \& Rizzo 1989).

\section{Results and analysis}

To provide meaningful results in physics, the typical values of the parameters of ice sheet and fluid are taken to be

$$
E=5 \mathrm{GPa}, \nu=0.3, \rho_{i}=922.5 \mathrm{~kg} / \mathrm{m}^{3}, h=1 \mathrm{~m}, \rho_{w}=1025 \mathrm{~kg} / \mathrm{m}^{3}, H=100 \mathrm{~m},
$$

which are the same as those in Squire \& Dixon (2000), and the considered wave length $\lambda_{0}=2 \pi / \kappa_{0}$ will vary from $10 \pi \mathrm{m}$ to $400 \pi \mathrm{m}$. The length scales of closed and open cracks are chosen to be similar to that in Li et al. (2018c) and to that in Porter \& Evans (2007) respectively. In the following text, numerical results will be provided in the 


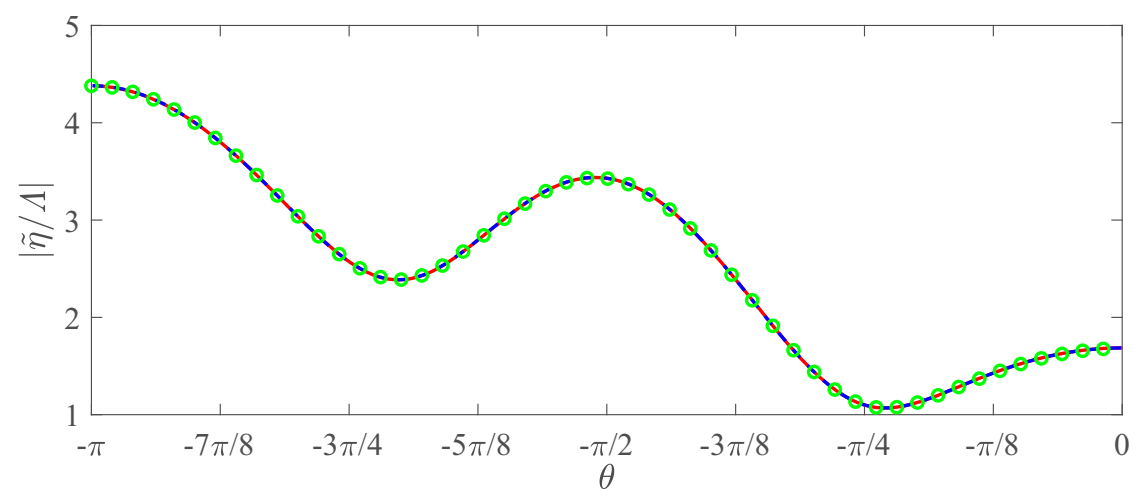

$(a)$

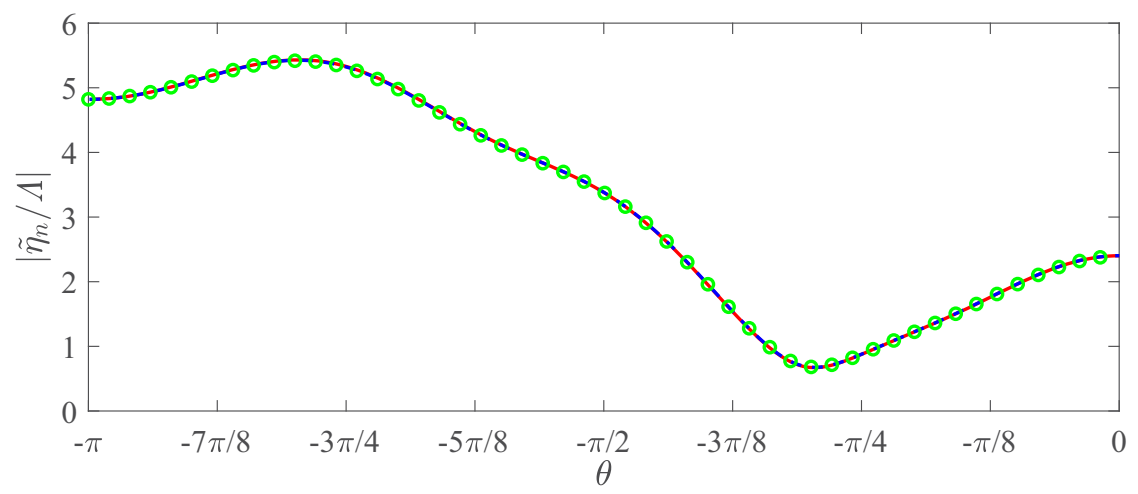

$(b)$

Figure 2. Modulus of the jump of displacement $\tilde{\eta} / \Lambda$ and that of slope $\tilde{\eta}_{n} / \Lambda$ along the crack with different $M_{i}$ and $T$ at $\kappa_{0}=3$ and $\beta=0$. Solid lines: $M_{i}=500$ and $T=100$; dashed lines: $M_{i}=1000$ and $T=200$; open circles: analytical solution from Li et al. $(2018 c)$.

dimensionless form, based on three basic parameters, or a characteristic length scale, the density of water $\rho_{w}$, and acceleration due to gravity $g=9.80 \mathrm{~m} / \mathrm{s}^{2}$. To conduct numerical computations, in addition to the discretization of cracks, the infinite series in (3.5) will be truncated at a finite number $T-3$ or the first $T$ terms will be kept. Higher accuracy can be achieved through smaller segments and more terms being kept in the eigenfunction expansions. It might be noticed that the computational cost very much depends on the number of segments $M=M_{1}+\cdots+M_{N}$ used to discretize the integrals (3.32) and (3.35) and the number of terms $T$ used to truncate the infinite series (3.36) and (3.37). Generally, the CPU time required to construct the coefficient matrix is proportional to square of $M$ and is to $T$ linearly. For the case studies here, $M_{i}=500$ and $T=100$ with $N$ up to 4 are found to be enough to provide convergent results for all the considered crack shapes and wave lengths. For $N=1$, the developed sequential FORTRAN 90 code WISPICE requires about $24.8 \mathrm{~s}$ to obtain the solution for each wave length in computer with CPU as i7 6700k. When they become very large, further refinement of the numerical method is needed, such as through domain decomposition method (Wu \& Eatock Taylor 1995). 


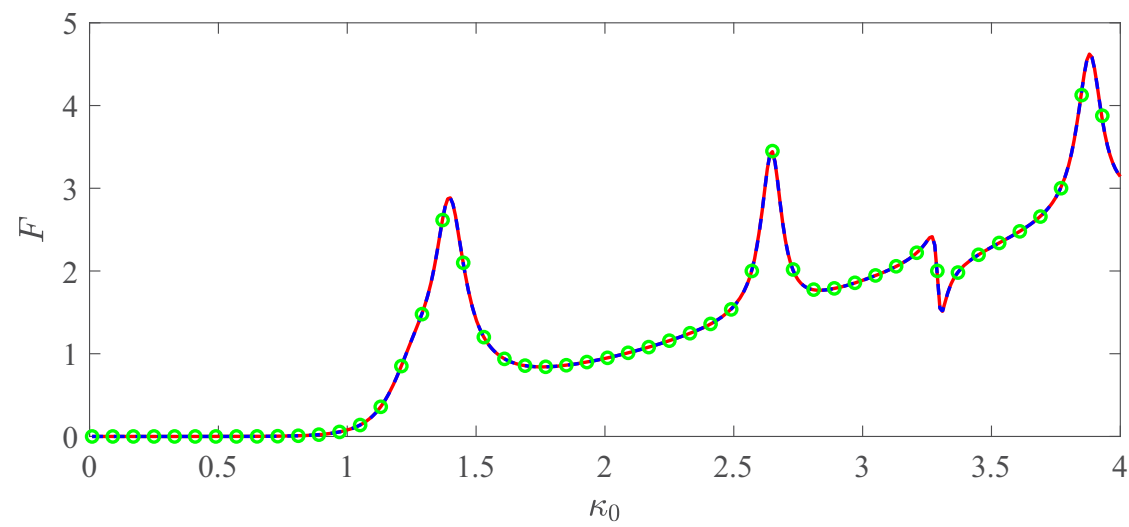

Figure 3. Scattered cross section $F$ against wave number $\kappa_{0}$ at $\beta=0$. Solid line: $F$ from (A 4); dashed line: $F$ from (A 9); open circles: analytical solution from Li et al. (2018c).

\subsection{Convergence study and verification}

Computations are first carried out for the wave diffraction by a circular crack with radius $a$ in an ice sheet. The case has been investigated by $\mathrm{Li}$ et al. (2018c) and an explicit solution was obtained. Here, $a=20 \mathrm{~m}$ is taken to be the characteristic length scale, and the same parameters as those in Li et al. (2018c) have been used, namely $\kappa_{0}=3$ and $\beta=0$. Figure 2 shows the modulus of the jump of displacement $\tilde{\eta}$ and that of slope $\tilde{\eta}_{n}$ across the crack against polar coordinate $\theta=\arctan (y / x)$, which are obtained through the kinematic condition on the ice sheet, or

$$
\tilde{\eta}=\frac{\tilde{D}}{\mathrm{i} \omega} \quad \text { and } \quad \tilde{\eta}_{n}=\frac{\tilde{S}}{\mathrm{i} \omega} .
$$

Due to symmetry of the problem, only the results for $\theta \in[-\pi, 0]$ are provided. It can be seen from the figure that there is no visible difference between the results obtained by $M_{i}=500, T=100$ and $M_{i}=1000, T=200$, indicating that the convergence has been achieved. Here, it may be noticed that the results are obtained through discretizing the crack into segments with equal length. From the figure, it can be also observed that the numerical results are in an excellent agreement with the analytical solutions from $\mathrm{Li}$ et al. (2018c), which validates the proposed solution procedure. Further verification of the solution procedure is carried out through the scattered cross section $F$ in (A 4), as shown in figure 3 against wave number $\kappa_{0}$. Numerical computation is carried out through taking $M_{i}=500$ and $T=100$. It can be seen from this figure that the results obtained by direct integration of $U(\theta)$ show a good agreement with those computed by equation (A 9), and both these two sets of results agree well with the analytical solutions. This again demonstrates the accuracy of the present solution procedure.

\subsection{Wave diffraction by a single closed crack}

In this and following sections, the ice thickness $h$ will be taken as the characteristic length scale, and $M_{i}=500$ and $T=100$ will be used for numerical computations, if it is not specifically specified. We first investigate the problem of flexural gravity wave interactions with a closed crack, and the effect of its shapes on the scattered wave field. For this purpose, closed cracks with elliptic shape are considered, i.e. defined as $(x / a)^{2}+(y / b)^{2}=1$, where $a$ and $b$ are the half axes in the $x$ and $y$ directions, respectively. To demonstrate how wave diffraction by the crack will be affected when its shape changes and also to get a better understanding of flow physics for this type of problem, three 


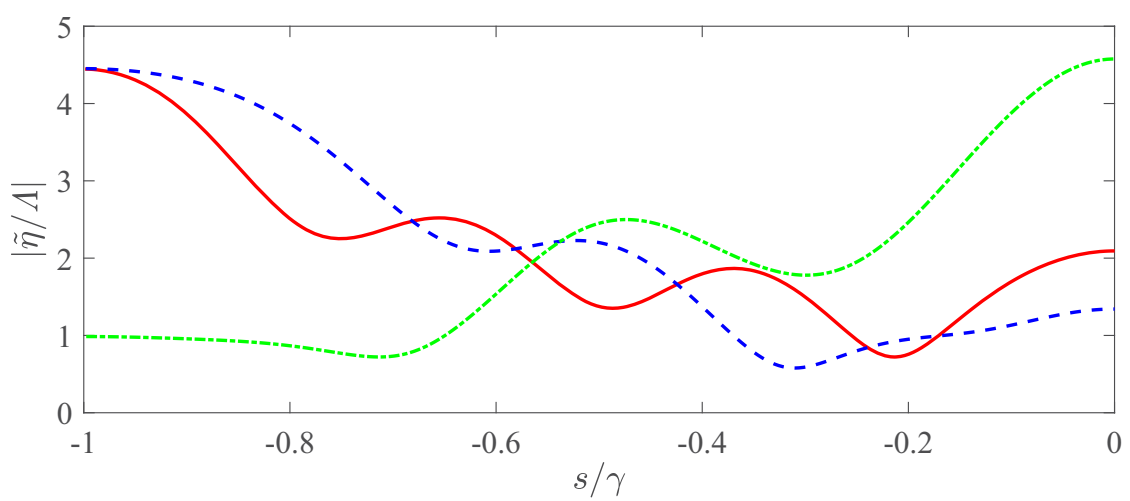

(a)

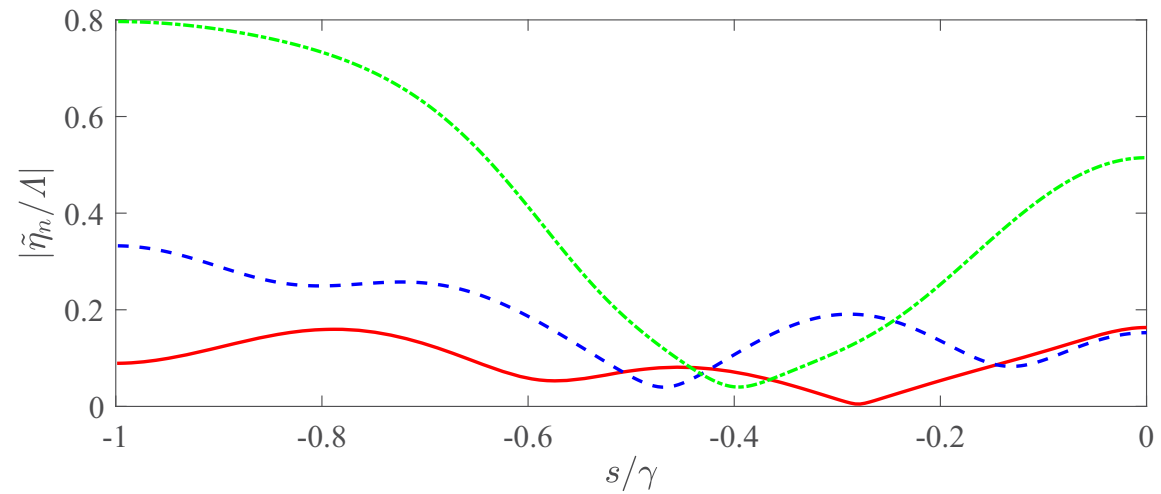

(b)

Figure 4. Modulus of the jump of displacement $\tilde{\eta} / \Lambda$ and that of slope $\tilde{\eta}_{n} / \Lambda$ along the elliptic crack with different $b / a$ at $\kappa_{0}=2 \pi / \sqrt{A}$ and $\beta=0(A=\pi a b=400 \pi)$. Solid lines: $b / a=0.5$; dashed lines: $b / a=1.0$; dash-dotted lines: $b / a=1.5$.

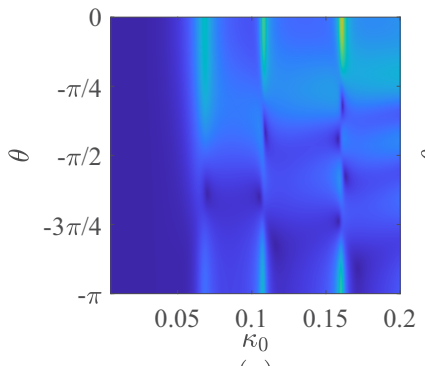

(a)

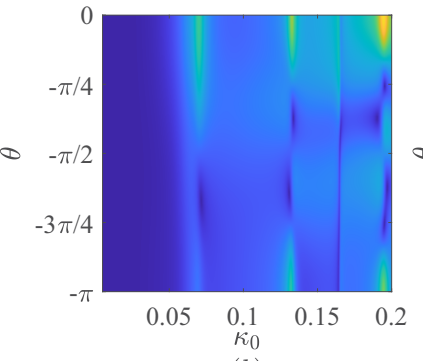

(b)

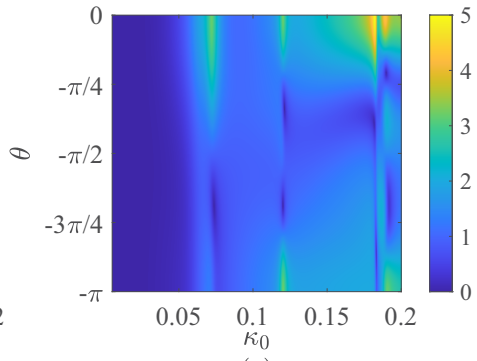

(c)

Figure 5. Modulus of the diffraction coefficient $U$ as a function of $\theta$ and $\kappa_{0}$ for elliptic cracks with different $b / a$ at $\beta=0(A=\pi a b=400 \pi)$. (a) $b / a=0.5 ;(b) b / a=1.0 ;(c) b / a=1.5$.

crack shapes are chosen, namely $b / a=0.5,1.0,1.5$ with its confined area kept fixed or $A=400 \pi$. For discretization, the nodes of the crack are distributed in the following form $x^{j}=a \cos \left[\frac{(2 j-M) \pi}{M}\right]$ and $y^{j}=b \sin \left[\frac{(2 j-M) \pi}{M}\right]$ with $j=0,1, \ldots, M-1$.

The jumps of displacement $\tilde{\eta}$ and slope $\tilde{\eta}_{n}$ along the crack are respectively shown in 


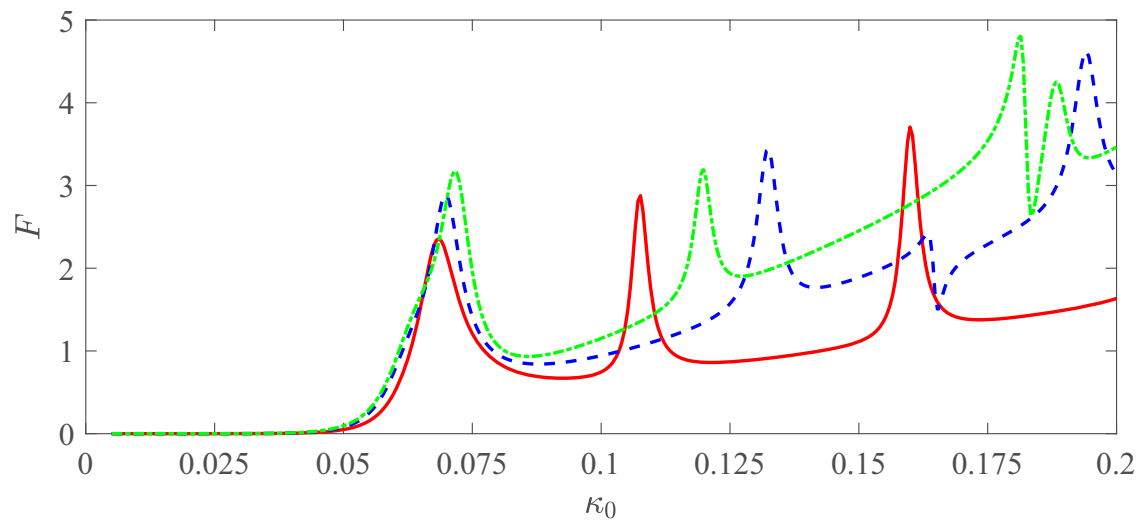

Figure 6 . Scattered cross section $F$ against wave number $\kappa_{0}$ for elliptic cracks with different $b / a$ at $\beta=0(A=\pi a b=400 \pi)$. Solid line: $b / a=0.5$; dashed line: $b / a=1.0$; dash-dotted line: $b / a=1.5$.

figure 4(a) and 4(b) against curvilinear coordinate $s / \gamma$, with the incident wave number taken to be $\kappa_{0}=2 \pi / \sqrt{A}$. The wave length corresponding to this $\kappa_{0}$ is equal to the characteristic scale of the crack or $\sqrt{A}$. Here, the incident wave angle is chosen as $\beta=0$, and thus only half of the $s$ range or $s / \gamma \in[-1,0]$ is plotted due to symmetry. It ought to be mentioned that the results given in this figure are convergent, namely $M_{i}=500$ and $T=100$ are found to be large enough and their larger values give graphically indistinguishable curves. It can be seen from this figure that the jumps of $\tilde{\eta}$ and $\tilde{\eta}_{n}$ at the crack are very much affected by the shape of the crack, although the areas enclosed by crack are the same. Typically, $\tilde{\eta}$ and $\tilde{\eta}_{n}$ will oscillate with $s / \gamma$, and the number of the oscillation and its peaks and troughs are different when the crack shape varies.

As given in equation (A 1), the far field diffracted wave field may be described by a diffraction coefficient $U(\theta)$, which can be obtained through an integral involving jumps $\tilde{D}$ and $\tilde{S}$ along the crack, as shown in (A 3). In figure $5, U$ is sketched through a contour plot as a function of polar coordinate $\theta$ and wave number $\kappa_{0}$. It can be seen from this figure that there is a strong variation of the far field diffracted waves for crack with different shapes. It can be also seen from figure 5 that when $\kappa_{0} \rightarrow 0$, there will be no diffracted wave by the crack or the ice sheet is equivalent to a continuous one. From mathematical aspect, when $\kappa_{0} \rightarrow 0$ or $\omega \rightarrow 0$ the ice sheet condition in (2.6) will become $\partial \phi / \partial z=0$. This indicates that the free edge condition in (2.7) at the crack and no concentrated force condition in (2.13) will be automatically satisfied, or the ice sheet will be "continuous". From physical aspect, when the wave length $\lambda_{0}=2 \pi / \kappa_{0}$ tends to infinity, the dynamic effect of wave motion will disappear, and so does the disturbance to the ice sheet, either with a crack or without. When $\kappa_{0}$ increases, the diffraction coefficient $U$ becomes more pronounced, and for a given $\kappa_{0}$ there is an obvious difference of the distribution of $U$ against $\theta$ for a crack with a different shape. A common feature is that they all oscillate against $\theta$, and a larger $\kappa_{0}$ will lead to more oscillatory results. This is because more wave cycles will be encountered by a given crack when the wave length decreases. It can be also seen from figure 5 that for all these three situations and various $\kappa_{0}$, a large part of the diffracted wave energy appears near the direction of the incident wave. From this figure, we can further observe that when $\theta$ is fixed, $U$ will oscillate with respect to $\kappa_{0}$. This can be more clearly seen from figure 6 which shows the 2D curves of scattered cross section $F$ against $\kappa_{0}$. For the specific range of $\kappa_{0}$, three large peaks of $F$ can be observed in addition to some local small peaks for different crack shapes. This is one of the typical 


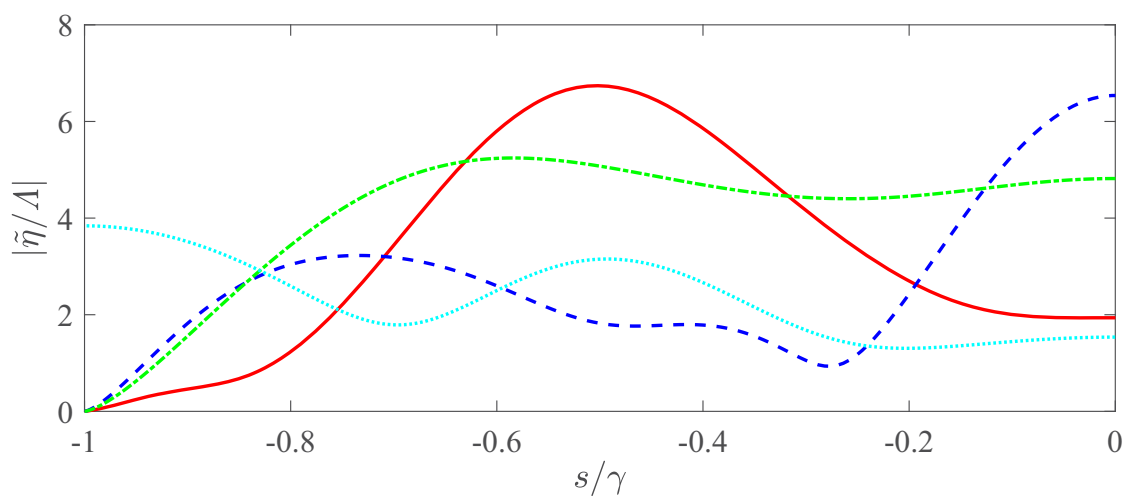

(a)

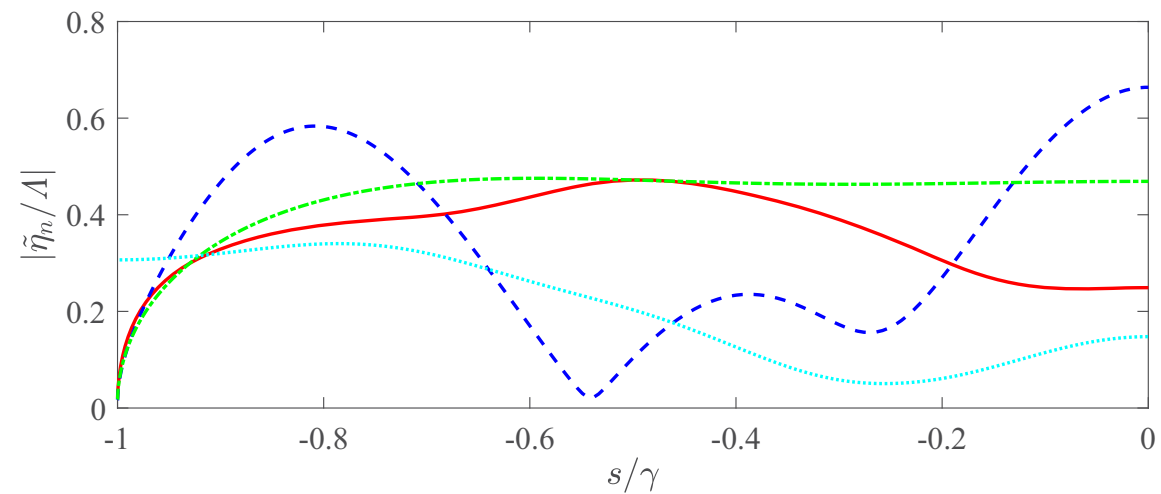

(b)

Figure 7 . Modulus of the jump of displacement $\tilde{\eta} / \Lambda$ and that of slope $\tilde{\eta}_{n} / \Lambda$ along circular cracks with different open angle $\vartheta$ at $\kappa_{0}=\pi / 20$ and $\beta=0(\gamma=20 \pi)$. Solid lines: $\vartheta=\pi / 20$; dashed lines: $\vartheta=\pi / 3$; dash-dotted lines: $\vartheta=39 \pi / 20$; dotted lines: closed circular crack.

features for a closed crack. It is well known that for a finite elastic plate in vacuum, after an initial disturbance of the plate, it will oscillate forever at its natural frequencies if the structural damping effect is ignored. The oscillation amplitude will tend to infinity if the frequency of the continuous exciting force is equal to one of the natural frequencies of the plate. However, for ice plate inside the crack, when there is fluid in its lower side, its oscillations will generate water waves. Part of energy will transmit through the crack and propagate to infinity, which provides a damping through wave radiation. The peaks at the natural frequencies which will be different from those in the vacuum may be very large instead of infinity. When the geometry of ice plate varies, its natural frequencies can be expected to be different (Smith et al. 2011). Therefore, for a closed crack with various shapes, its near natural frequencies will be different or the large peaks will occur at different wave numbers, as shown in figure 6.

\subsection{Wave diffraction by a single open crack}

Investigations now move to the question of how differently open cracks will affect the diffracted wave fields. To do this, we consider circular cracks with different open angles, i.e. $x=a \cos \theta$ and $y=a \sin \theta$ with $\theta \in[-\bar{\theta},+\bar{\theta}]$. Here $\vartheta=2(\pi-\bar{\theta})$ is the open angle. Three different crack are chosen, i.e. $\vartheta=\pi / 20, \pi / 3$ and $39 \pi / 20$, with their length fixed as $40 \pi$. It should be noticed that the corresponding radius of these three cracks are 


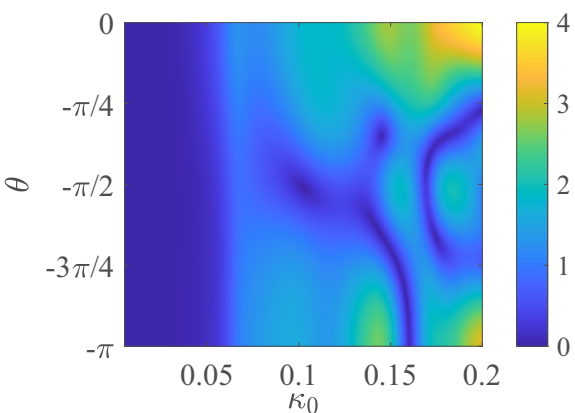

$(a)$

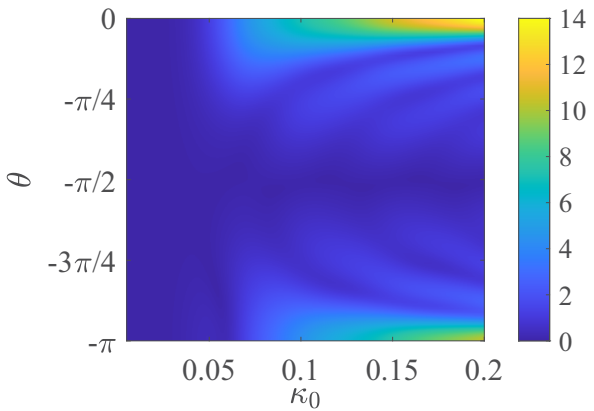

(c)

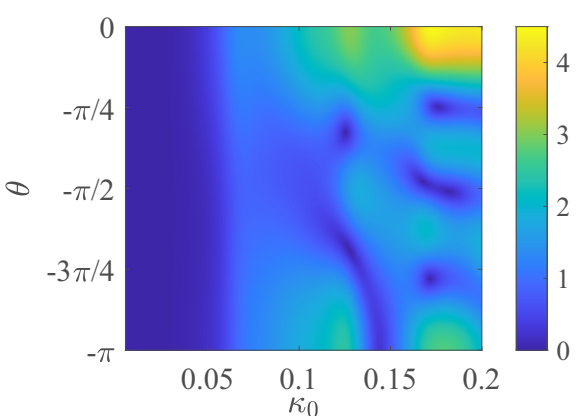

(b)

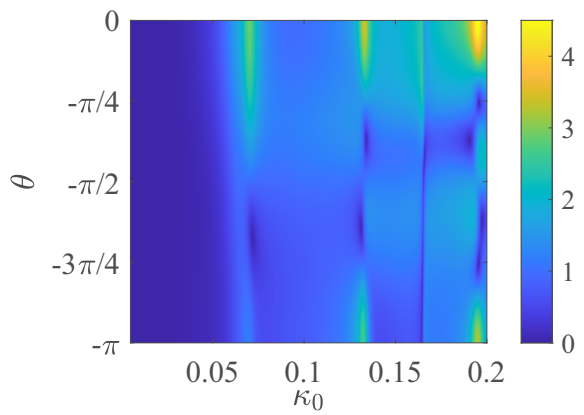

$(d)$

Figure 8. Modulus of the diffraction coefficient $U$ as a function of $\theta$ and $\kappa_{0}$ for circular cracks with different open angle $\vartheta$ at $\beta=0(\gamma=20 \pi)$. (a) $\vartheta=\pi / 20 ;(b) \vartheta=\pi / 3 ;(c) \vartheta=39 \pi / 20 ;(d)$ closed circular crack.

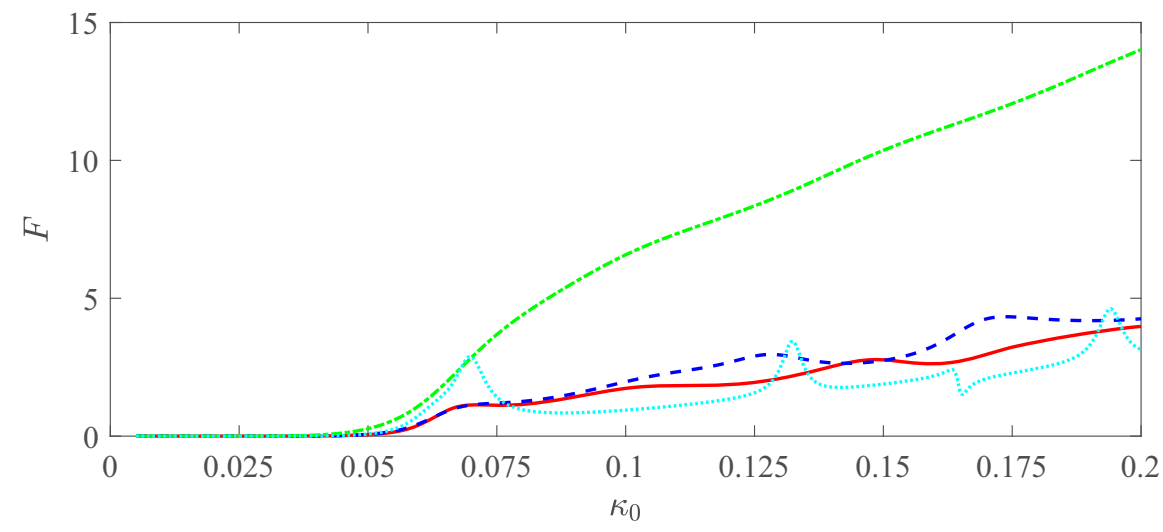

Figure 9. Scattered cross section $F$ against wave number $\kappa_{0}$ for circular cracks with different open angle $\vartheta$ at $\beta=0(\gamma=20 \pi)$. Solid line: $\vartheta=\pi / 20$; dashed line: $\vartheta=\pi / 3$; dash-dotted line: $\vartheta=39 \pi / 20$; dotted line: closed circular crack.

$800 / 39,24$ and 800 respectively. The incident wave number is chosen as $\kappa_{0}=\pi / 20$, or the incident wave length $\lambda_{0}=2 \pi / \kappa_{0}=40$ which is the diameter of the case with $\vartheta=0$, and the incident wave angle is chosen as $\beta=0$. From equation (A 8) in Appendix, we have the asymptotic behaviours $\tilde{D}=O\left(s^{\alpha}\right)$ and $\tilde{S}=O\left(s^{\alpha-1}\right)$ near a tip of the open crack, with $\alpha \geqslant 3 / 2$, where $s$ is the distance measured from the tip. From this, it can be expected that their partial derivatives $\partial^{2} \tilde{D} / \partial s^{2}$ and $\partial \tilde{S} / \partial s$ involved in the integral equations (3.32) and (3.35) could vary very sharply near the tips of the crack. Therefore, 
to accelerate convergence of the results in the computations, the nodes are distributed in a cosine form along the crack, or

$$
s^{j}=-\gamma+\gamma[1-\cos (j \pi / M)] \quad \text { with } j=0,1, \ldots, M .
$$

The jumps of displacement and slope at the crack are shown in figure 7 against curvilinear coordinate $s / \gamma$. As a comparison, the results for a closed circular crack are also provided. It can be seen that the jumps due to the diffracted wave at the cracks with the same length but different curvatures differ from each other significantly. Particularly, the results of larger curvature $(39 / 800$ and $1 / 24)$ are much more oscillatory against $s / \gamma$ than those of very small curvature $(1 / 800)$ in the figure. The small curvature case is closer to that of a straight line crack parallel to the $y$-axis. When its length is very large and approaches infinity, the problem tends to a $2 \mathrm{D}$ one, and $\tilde{\eta}$ and $\tilde{\eta}_{n}$ will be constant along the crack or their variations will be zero. From figure 7 , it is also interesting to see that when the opening of a crack is small and its two ends tend to merge to form a closed crack, the diffracted wave field of the former does not tend to that of the latter, as can be seen through the solid lines and dashed lines. In fact, although the open crack has nearly become a closed crack, the physics is still different. For the latter one, the inside ice sheet is fully separated from the outside ice sheet by the crack, or there is no direct connection between the two ice sheets, and their interactions are through the fluid. However, for an open crack, the ice sheets on both sides of the crack remain fully connected, and there will always be a direct interaction between their motions. When the open angle tend to zero, the connection will become a point at which the jumps $\tilde{\eta}$ and $\tilde{\eta}_{n}$ should still be zero, as shown in figure 7 , which is different from that of the closed one.

The effect of open crack shape on the diffracted wave in far field is demonstrated in figure 8, through the diffraction coefficient $U$ in contour plot as a function of $\theta$ and $\kappa_{0}$. It can be observed from this figure that $U$ tends to be zero as $\kappa_{0} \rightarrow 0$ for the reason explained in $\S 5.2$. When $\kappa_{0}$ increases, the diffracted wave become more obvious. From the contour plot, we have that the diffracted wave fields differ from each other significantly for cracks with the same length but different shapes. However, similar to the closed crack case, most of the diffracted wave will propagate in the direction of incident wave $\beta$ for all situations. For the crack with a very small curvature, there is also a large part of diffracted wave in direction of $-\beta$, as shown in figure $8(c)$. This is similar to the waves normally incident to an infinitely straight line crack of zero curvature, in which waves will transmit to the direction 0 and be reflected back to the direction $\pi$. As discussed in the above section, there will be near resonant motions when the crack is closed, which will lead to large amplitude diffracted waves or $U(\theta)$. When the crack is open, no similar resonant behaviors can be observed, even for a very small open angle. This can be seen from the comparisons of figure $8(a)$ with $8(d)$, and more clearly be observed from figure 9 which provides the variations of scattered cross section coefficient $F$ against $\kappa_{0}$. From the contour plot in figure 8 , we also have that when $\kappa_{0}$ is fixed, $U$ will oscillate with $\theta$. As $\kappa_{0} \rightarrow 0.2$, there will be more cycles of $U$ with respect to $\theta$ for a moderate open angle in figure $8(b)$.

\subsection{Wave diffraction by multiple cracks}

The developed methodology in this paper can be applied to any crack shapes and arrangements. Here, we will consider a few cases of wave diffraction by multiple cracks. The first case we consider is two straight line cracks with the same finite length of $2 \gamma_{i}=40 \pi, i=1,2$. One is parallel to the $y$-axis with its start and end points at $\left(x_{s}^{1}, y_{s}^{1}\right)=(-2.5,-20 \pi)$ and $\left(x_{e}^{1}, y_{e}^{1}\right)=(-2.5,20 \pi)$ respectively. For the other crack, its start point is fixed at $\left(x_{s}^{2}, y_{s}^{2}\right)=(2.5,-20 \pi)$, but its angle with respect to $x$-axis 


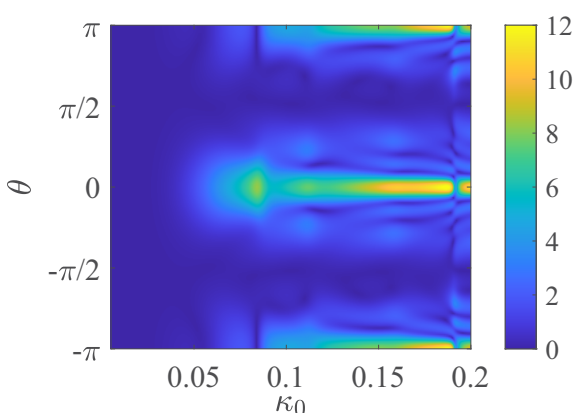

(a)

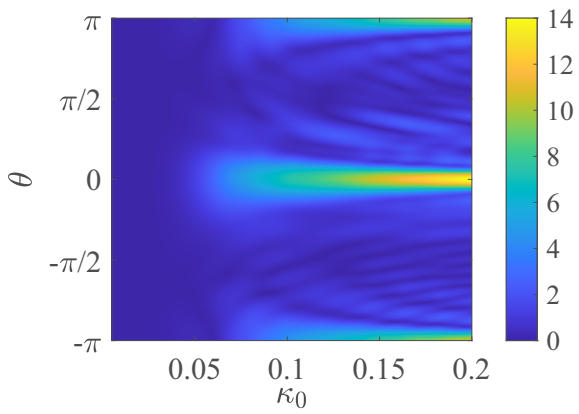

(c)

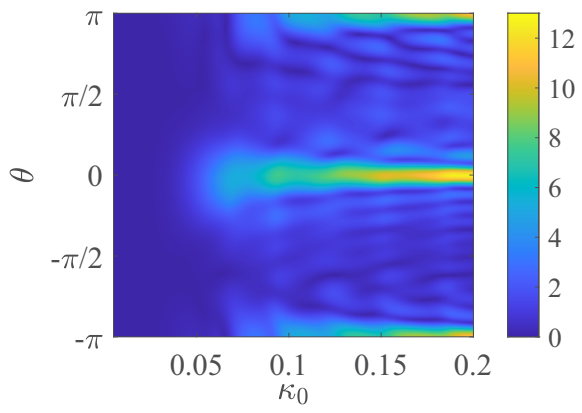

(b)

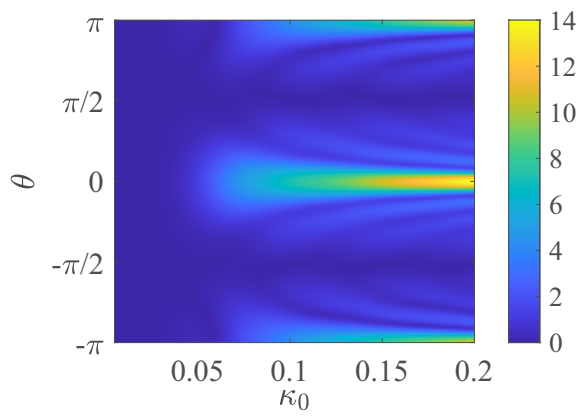

(d)

Figure 10. Modulus of the diffraction coefficient $U$ as a function of $\theta$ and $\kappa_{0}$ for two straight line cracks with different arrangements at $\beta=0$. (a) $\tau=\pi / 2 ;(b) \tau=\pi / 4 ;(c) \tau=0 ;(d)$ single crack.

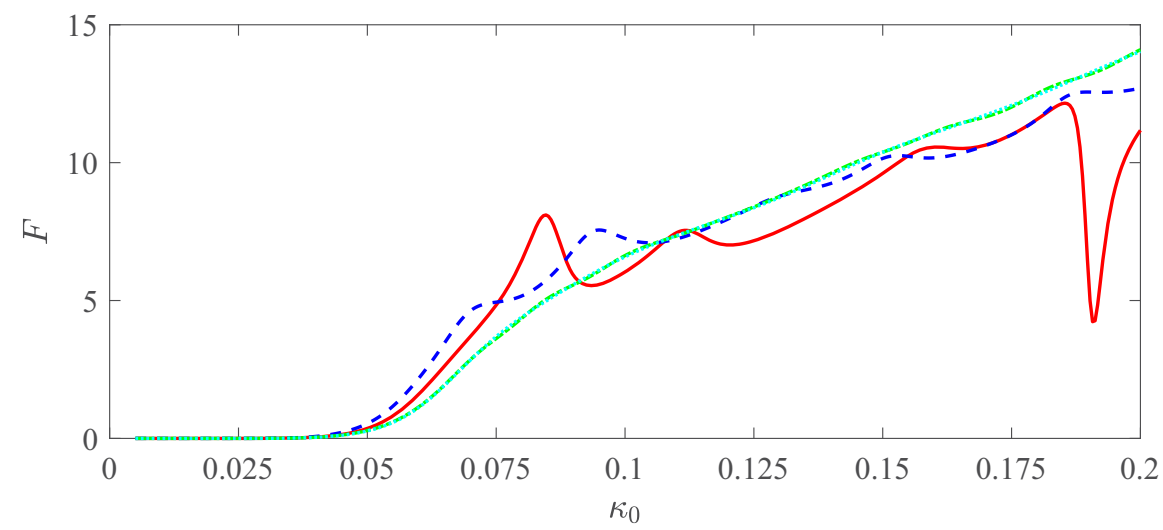

FiguRe 11. Scattered cross section $F$ against wave number $\kappa_{0}$ for two straight line cracks with different arrangements at $\beta=0$. Solid line: $\tau=\pi / 2$; dashed line: $\tau=\pi / 4$; dash-dotted line: $\tau=0$; dotted line: single crack.

changes, namely $\tau=\pi / 2, \pi / 4$ and 0 . Both of these two cracks are discretized with nodes distributed in the cosine form in (5.4), which provides a very finer mesh near the tips of crack.

Figure 10 provides the contour plot of diffraction coefficient $U$ as a function of polar coordinate $\theta$ and wave number $\kappa_{0}$, for two straight line cracks with different arrangements. As a comparison, the results for a single crack or with the second crack above being removed are also provided. Here, the incident wave angle is taken as $\beta=0$. Different 


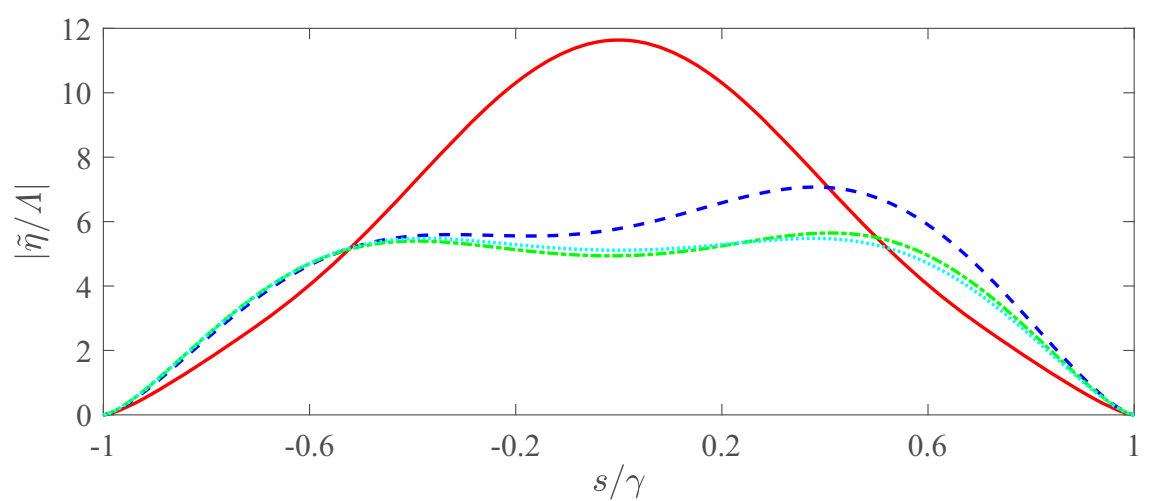

(a)

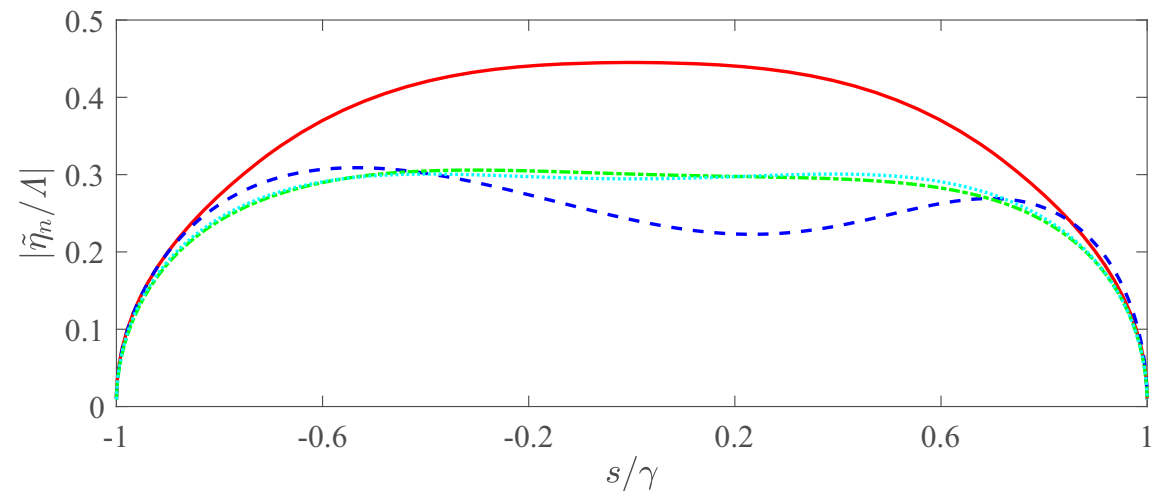

(b)

Figure 12. Modulus of the jump of displacement $\tilde{\eta} / \Lambda$ and that of slope $\tilde{\eta}_{n} / \Lambda$ along the left crack for different arrangements of the right crack at $\kappa_{0}=0.1$ and $\beta=0$. Solid lines: $\tau=\pi / 2$; dashed lines: $\tau=\pi / 4$; dash-dotted lines: $\tau=0$; dotted lines: single crack.

from the case in $§ 5.3$, since the cracks are not arranged symmetrically about $y=0$, the obtained results are asymmetric, as shown in the figure. From the figure, it can be seen that the diffracted wave field varies differently for cracks with different arrangements, although they all tend to be zero when $\kappa_{0} \rightarrow 0$ for the reason discussed in $\S 5.2$. When the two cracks are closely arranged, it is expected that there will be stronger interactions between waves generated by the cracks, as can be observed in figure $10(a)$ and $10(b)$. This is clearer in figure 11, which shows the variation of the scattered cross section $F$ against $\kappa_{0}$. For two cracks parallel to each other or $\tau=\pi / 2$, it resembles a $2 \mathrm{D}$ problem. There is much strong variation with $\kappa_{0}$ in this case. When the right crack is arranged parallel to the $x$-axis, or $\tau=0$, the diffracted wave in the far field is very similar to that by a single crack. In figure 11, the dash-dotted line and dotted line respectively for these two cases are nearly overlapping each other. In fact, for a crack parallel to $x$-axis, by noticing $\partial \Theta / \partial s=0, \partial / \partial s=\partial / \partial x$ and $\partial / \partial n=-\partial / \partial y$, the operators $\mathcal{B}$ and $\mathcal{S}$ in equations (2.11) and (2.12) can be rewritten as

$$
\begin{gathered}
\mathcal{B}=\frac{\partial^{2}}{\partial y^{2}}+\nu \frac{\partial^{2}}{\partial x^{2}} \\
\mathcal{S}=-\frac{\partial}{\partial y}\left[\frac{\partial^{2}}{\partial y^{2}}+(2-\nu) \frac{\partial^{2}}{\partial x^{2}}\right] .
\end{gathered}
$$


For an incident wave with $\beta=0$ the right hand sides of equations (3.36) and (3.37) become

$$
\mathcal{B}\left(\frac{\partial \phi_{I}}{\partial z}\right)=\nu \frac{\partial^{3} \phi_{I}}{\partial x^{2} \partial z} \quad \text { and } \quad \mathcal{S}\left(\frac{\partial \phi_{I}}{\partial z}\right)=0
$$

which means that only the second term in (5.5) has a contribution to the results. Then, it can be expected that there will be more diffracted waves for $\beta=\pi / 2$ than those for $\beta=0$. This feature has also been shown in the numerical results of Porter \& Evans (2007), where they considered only the straight and parallel crack lines. For the case in figure $10(c)$, incident wave propagates along the $x$-axis. It will arrive with normal incident angle at the left crack first, and generate a diffracted wave which will mainly propagate along and opposite to the positive $x$-axis, as demonstrated in $\S 5.3$. The new wave field can be seen as an incident wave for the other crack. When it arrives at the right crack which is parallel to $x$-axis, the generated diffracted wave will be very small, as the direction of the main component of the incident wave field is parallel to $x$-axis. This indicates that the results for two cracks will be similar to those for a single crack in figure $10(\mathrm{c})$.

In figure 12, the jumps of displacement and slope at the left crack are shown for various arrangements of the right crack. The shapes and arrangements of the cracks as well as the incident wave angle are the same as those in figure 10, while the incident wave number is taken to be $\kappa_{0}=0.1$. As can be expected, the jumps of all situations tend to be zero as the point approaches to the tips of crack. Similar to $U$ or $F$ in figures 10 and 11, from figure 12, it can be observed that the jumps of displacement and slope along the crack for $\tau=0$ are also close to those for a single crack.

The cracks of a uniform ice sheet formed by the excitation of a unidirectional regular wave might have a simple shape. Due to the random nature of the ocean waves, the cracks in Polar regions may be generally complex, which can be either open, closed, or the mixture of them. This can be partly reflected by the photos taken on site as well as in the laboratory (Kohout et al. 2016; Herman et al. 2018). Also, when there is a high concentration of ice floes with very narrow gaps between the floes, this could be equivalently treated as a crack problem mathematically, as noted by Squire \& Dixon (2001a). The shapes of these narrow gaps could be highly complex (e.g. Fig. 2, Herman et al. 2018). Therefore, in our final case we consider a more general situation, or the flexural gravity wave interactions with multiple mixed closed and open cracks, i.e. two closed circular cracks with radius $a$ as 20 and centre $(x, y)$ respectively at $(-40,0)$ and $(20,-20)$, and two straight line cracks parallel to $y$-axis with length $2 \gamma_{i}$ as 40 and centre $(x, y)$ respectively at $(-10,20)$ and $(50,0)$. Here, oblique incident wave with $\beta=\pi / 4$ is considered.

Figure 13 shows the jumps of displacement $\tilde{\eta}$ and slope $\tilde{\eta}_{n}$ along the cracks against curvilinear coordinate $s / \gamma_{i}$. Here, the incident wave number is taken to be $\kappa_{0}=\pi / 20$, and the corresponding wave length is equal to diameter of the circular crack or length of the straight line crack. Convergence with respect to $M_{i}$ and $T$ has been verified, and the energy conservation relationship in equation (A 9) has been used as a partial check. It can be observed from the figure that for the open cracks both $\tilde{\eta}$ and $\tilde{\eta}_{n}$ will tend to be zero as $s \rightarrow \pm \gamma_{i}$, as expected from the physics. For the specific wave length or $\kappa_{0}$, it can be seen that $\tilde{\eta}$ and $\tilde{\eta}_{n}$ will oscillate with $s / \gamma_{i}$, and more peaks and troughs appear for a closed circular crack than those for a straight line crack.

In figure 14, contour plots are shown for the diffraction coefficient $U$ as a function of $\theta$ and $\kappa_{0}$. It can be observed that similar to the single crack case, the wave energy is mainly diffracted to the direction of the wave incidence. The diffraction coefficient for 


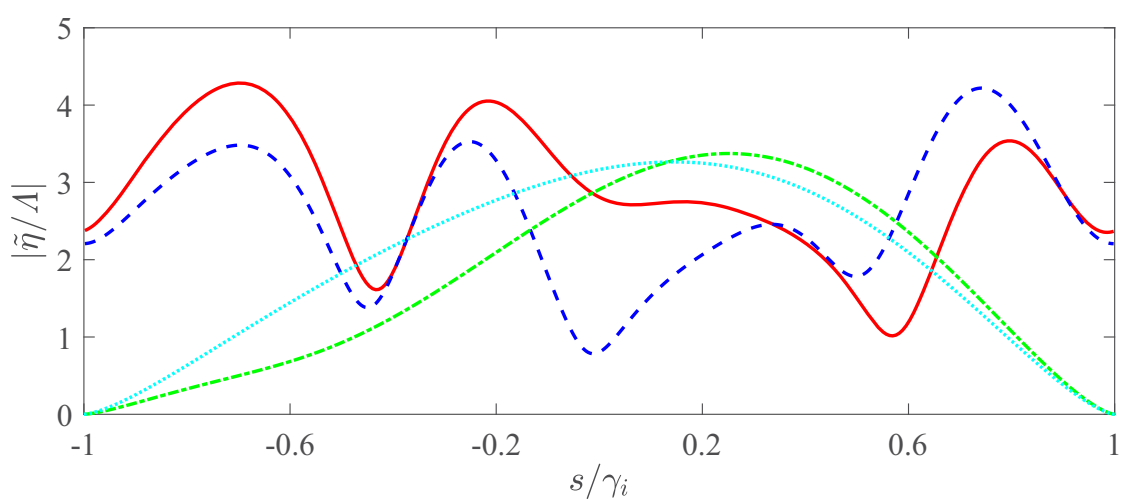

(a)

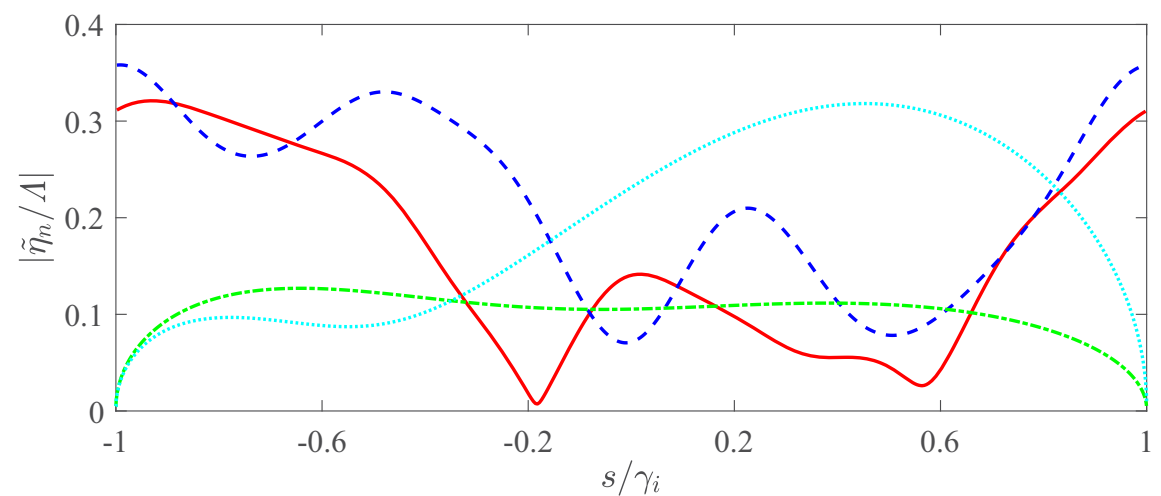

(b)

Figure 13. Modulus of the jump of displacement $\tilde{\eta} / \Lambda$ and that of slope $\tilde{\eta}_{n} / \Lambda$ against curvilinear coordinate $s / \gamma_{i}$ for multiple mixed closed and open cracks at $\kappa_{0}=\pi / 20$ and $\beta=\pi / 4$. Solid lines: circular crack at $(-40,0)$; dashed lines: circular crack at $(20,-20)$; dash-dotted lines: straight line crack at $(-10,20)$; dotted lines: straight line crack at $(50,0)$.

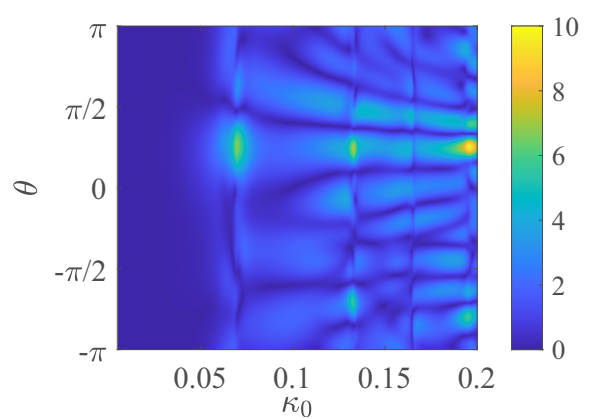

(a)

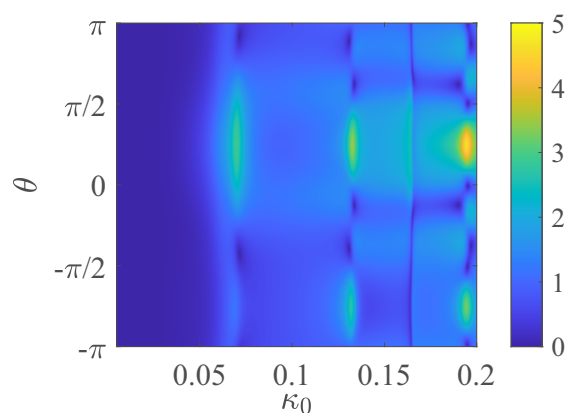

(b)

Figure 14. Modulus of the diffraction coefficient $U$ as a function of $\theta$ and $\kappa_{0}$ at $\beta=\pi / 4$. (a) multiple mixed closed and open cracks; $(b)$ single closed circular crack.

a single circular closed crack is also shown in figure 14. It is interesting to see that the wave number $\kappa_{0}$ corresponding to the large peaks in figure 14(a) for multiple cracks are similar to those in figure 14(b) for a single crack, although the value of the former case is generally larger than the latter case. This can be more clearly observed from figure 15, 


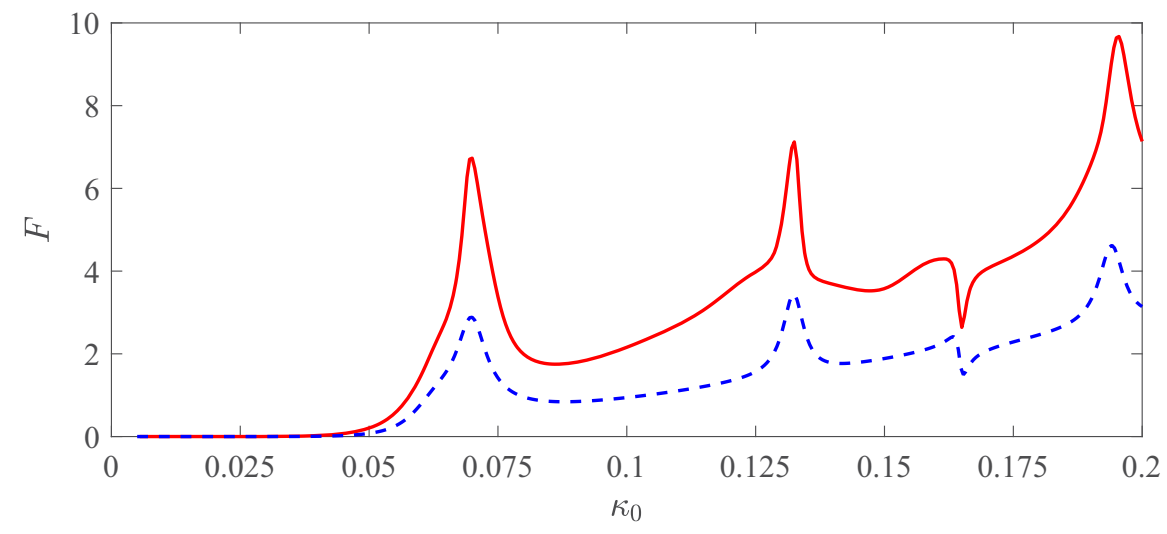

FIgURE 15. Scattered cross section $F$ against wave number $\kappa_{0}$ at $\beta=\pi / 4$. Solid line: multiple mixed closed and open cracks; dashed line: single closed circular crack.

which shows the scattered cross section $F$ against $\kappa_{0}$. Here, three large peaks appear within the range calculated. As discussed in $\S 5.2$, for a closed crack there will be a near resonant wave motions at a set of discrete $\kappa_{0}$. At these $\kappa_{0}$, the amplitude of the diffracted wave can be very large. For multiple cracks, there will be interactions between the waves due to different cracks. Thus, the wave numbers at which large motion may occur may not be exactly the same as those $\kappa_{0}$ for an individual crack. However, in many cases, these wave numbers are still close to $\kappa_{0}$. This is reflected by the results in figure 15 .

\section{Conclusions}

The problem of flexural gravity wave interactions with multiple cracks in ice sheet of infinite extent has been solved, in which both the shape and location of the cracks can be arbitrary, and the individual crack can be either open or closed. The problem is first transformed into an infinite number of coupled two dimensional problems in horizontal plane. Each two dimensional problem is converted into an integral equation along the cracks. By writing the potential in a series in the vertical direction and adopting an orthogonal inner product, all the required conditions including those at the crack are satisfied. The integral differential equations are solved using the boundary element method together with the finite difference scheme for the high derivatives along the crack.

For the interaction of wave with a closed crack, the jumps of displacement $\tilde{\eta}$ and slope $\tilde{\eta}_{n}$ are generally smooth along the crack length. However, there are several cycles of oscillations with peaks and troughs, and the amplitude of the oscillation is not a constant. For a closed crack, the enclosed ice sheet becomes detached from the external one. For an ice plate in vacuum, it will oscillate forever at its natural frequencies after an initial disturbance, if there is no structure damping. When the excitation force has a component at one of the natural frequencies or at resonance, the motion amplitude can be infinite. When the ice plate is on water surface, similar resonant behaviour can occur. However, the motion amplitude will not be infinite because of wave radiation damping. Instead, the motion will show large amplitude as reflected by the variation of the diffraction coefficient $U(\theta)$ with $\kappa_{0}$.

For an open crack, the variations of displacement and slope jumps are less oscillatory. Both jumps tend to zero at the tip of the crack. However, the slope jump changes very rapidly near the tip and its high derivative is singular at the tip. This singularity has to be treated analytically in the integral equation and numerically in the discretization, 
as has been done successful in this work. The displacement and slope jumps are not as oscillatory as those of a closed crack. It is interesting to see even when the two tips of the open crack are very close, the results do not tend to those of a closed one. A single point connection between the internal and external ice sheets make the physics very different.

When there are multiple cracks, both the crack shapes and their arrangements will have effects on the diffracted waves. Interactions between cracks are much stronger in the local field flow than that in the far field flow. This is mainly due to the fact that the evanescent waves of each crack have strong effects only locally. For a straight line crack, when it is parallel to the local wave direction, its effect to the total flow is small. Its removal does not have much effect on the overall diffraction coefficient.

The developed methodology can be also used to solve flexural gravity wave diffractions by cracks with corners. In such a case no concentrated force condition in equation (2.13) should be imposed at the corners. However, the method is limited to the ice sheet with constant physical properties, and the analysis is within the scope of the linear velocity potential theory.

\section{Acknowledgements}

This work is supported by Lloyd's Register Foundation, to which the authors are most grateful. Lloyd's Register Foundation helps to protect life and property by supporting engineering-related education, public engagement, and the application of research. This work is also supported by the National Natural Science Foundation of China (Grant No. 51709131 and 51879123)

\section{Declaration of interests}

The authors report no conflict of interest.

\section{Appendix A. Diffracted wave in far field and the scattered cross section}

After $\tilde{D}$ and $\tilde{S}$ having been found, similar to Li et al. (2018c) for wave diffraction by a circular crack, we may write the diffracted velocity potential $\phi_{D}$ in the far field as

$$
\lim _{r \rightarrow+\infty} \phi_{D}=\sqrt{\frac{2}{\pi \kappa_{0} r}} \mathrm{e}^{-\mathrm{i}\left(\kappa_{0} r-\pi / 4\right)} I U(\theta) \psi_{0}(z) / \tanh \left(\kappa_{0} H\right),
$$

where $x=r \cos \theta$ and $y=r \sin \theta, \xi=r_{0} \cos \theta_{0}$ and $\eta=r_{0} \sin \theta_{0}$, and the diffraction coefficient $U(\theta)$ represents the scattered wave energy distribution along the circumferential direction. Substituting (3.32) into (3.5) and noticing

$$
H_{0}^{(2)}\left(\kappa_{m} R\right)=\sqrt{\frac{2}{\pi \kappa_{m} r}} \mathrm{e}^{-\mathrm{i}\left(\kappa_{m} r-\pi / 4\right)} \mathrm{e}^{\mathrm{i} \kappa_{m} r_{0} \cos \left(\theta-\theta_{0}\right)} \quad \text { as } \quad r \rightarrow+\infty,
$$

we have

$$
\begin{aligned}
U(\theta)= & \frac{\pi \chi_{0}}{2 \mathrm{i} I} \tanh \left(\kappa_{0} H\right) \sum_{i=1}^{N} \int_{\Gamma_{i}}\left\{\left[\frac{\partial G_{\infty}}{\partial s_{q}} \nu_{0} \frac{\partial \Theta}{\partial s} \frac{\partial}{\partial s}-\frac{\partial G_{\infty}}{\partial n_{q}}\left(\kappa_{0}^{2}-\nu_{0} \frac{\partial^{2}}{\partial s^{2}}\right)\right] \tilde{D}\right. \\
& \left.+\left(G_{\infty} \kappa_{0}^{2}+\frac{\partial G_{\infty}}{\partial n_{q}} \nu_{0} \frac{\partial \Theta}{\partial s}-\frac{\partial G_{\infty}}{\partial s_{q}} \nu_{0} \frac{\partial}{\partial s}\right) \tilde{S}\right\} \mathrm{d} s_{q},
\end{aligned}
$$


where $G_{\infty}=\exp \left[\mathrm{i} \kappa_{0} r_{0} \cos \left(\theta-\theta_{0}\right)\right]$. Then the scattered cross section $F$ can be defined as follows

$$
F=\frac{1}{2 \pi} \int_{-\pi}^{+\pi}|U(\theta)|^{2} \mathrm{~d} \theta
$$

which is a measure of the total scattered energy.

It may be noticed that an energy conservation relationship for $F$ can be also derived. Applying Green's identity to $\phi$ and its complex conjugation $\phi^{*}$, we have

$$
\int_{S}\left(\phi \frac{\partial \phi^{*}}{\partial n}-\phi^{*} \frac{\partial \phi}{\partial n}\right) \mathrm{d} s=0
$$

where $S$ is comprised of sea bed $S_{H}$, ice sheet $S_{i}$ and a cylindrical surface at infinity $S_{\infty}$. Invoking the corresponding boundary conditions together with the Gauss theorem, we have

$$
\begin{aligned}
& I_{i}+\int_{S_{\infty}}\left(\phi \frac{\partial \phi^{*}}{\partial n}-\phi^{*} \frac{\partial \phi}{\partial n}\right) \mathrm{d} s \\
& +\frac{L}{\rho_{w} \omega^{2}} \int_{\Gamma_{\infty}}\left[\frac{\partial \phi^{*}}{\partial z} \frac{\partial \nabla^{2}}{\partial n} \frac{\partial \phi}{\partial z}-\frac{\partial \phi}{\partial z} \frac{\partial \nabla^{2}}{\partial n} \frac{\partial \phi^{*}}{\partial z}+\frac{\partial^{2} \phi}{\partial n \partial z} \nabla^{2} \frac{\partial \phi^{*}}{\partial z}-\frac{\partial^{2} \phi^{*}}{\partial n \partial z} \nabla^{2} \frac{\partial \phi}{\partial z}\right]_{z=0} \mathrm{~d} s=0,
\end{aligned}
$$

where

$$
I_{i}=\frac{2 \mathrm{i} \nu_{0} L}{\rho_{w} \omega^{2}} \sum_{i=1}^{N}\left\{\operatorname{Im}\left[D\left(\frac{\partial S^{*}}{\partial s}-\frac{\partial \Theta}{\partial s} \frac{\partial D^{*}}{\partial s}\right)+\frac{\partial D^{*}}{\partial s} S\right]_{-\gamma_{i}}^{+\gamma_{i}}\right\}_{+}^{-},
$$

which is due to the integral over $S_{i}$. The crack conditions in (2.7) together with integration by parts with respect to $s$ have been used in (A 7). For a closed crack, $I_{i}$ in (A 7 ) is zero explicitly. Based on the argument of the energy density at the crack tips, we have (Norris \& Wang 1994)

$$
D=O\left[\left(\gamma_{i} \mp s\right)^{\alpha}\right] \quad \text { and } \quad S=O\left[\left(\gamma_{i} \mp s\right)^{\alpha-1}\right], \quad\left(s \rightarrow \pm \gamma_{i}, z=0\right),
$$

where $\alpha \geqslant 3 / 2$. Thus, for an open crack, by noticing the asymptotic expressions of $D$ and $S$ in (A 8), $I_{i}$ in (A 7) is also zero. Then following the similar procedure in Li et al. (2018c) for a closed circular crack, we have

$$
F=-\operatorname{Re}[U(\beta)],
$$

which can be used as a partial check of the accuracy of the numerical results.

\section{REFERENCES}

Abramowitz, M. \& Stegun, I. A. 1965 Handbook of mathematical functions. New York: Dover press.

Balmforth, N. J. \& Craster, R. V. 1999 Ocean waves and ice sheets. Journal of Fluid Mechanics 395, 89-124.

BArrett, M. D. \& Squire, V. A. 1996 Ice-coupled wave propagation across an abrupt change in ice rigidity, density, or thickness. Journal of Geophysical Research Oceans 101 (C9), 20825-20832.

Bennetts, L. G. \& Squire, V. A. 2012 On the calculation of an attenuation coefficient for transects of ice-covered ocean. Proceedings of the Royal Society A468 (2137), 136-162.

Bennetts, L. G. \& Williams, T. D. 2010 Wave scattering by ice floes and polynyas of arbitrary shape. Journal of Fluid Mechanics 662, 5-35.

Burden, R. L. \& Faires, J. D. 2010 Numerical analysis. Boston: Brooks Cole. 
Chung, H. \& Fox, C. 2002 Calculation of wave-ice interaction using the wiener-hopf technique. New Zealand Journal of Mathematics 31 (1), 1-18.

Chung, H. \& Linton, C. M. 2005 Reflection and transmission of waves across a gap between two semi-infinite elastic plates on water. Quarterly Journal of Mechanics and Applied Mathematics $\mathbf{5 8}$ (1), 1-15.

Eliasson, K., Ulfarsson, G. F., Valsson, T. \& Gardarsson, S. M. 2017 Identification of development areas in a warming arctic with respect to natural resources, transportation, protected areas, and geography. Futures 85, 14-29.

Evans, D. V. \& Davies, T. V. 1968 Wave-ice interaction. Report. Davidson Laboratory, Stevens Institute of Technology.

Evans, D. V. \& Porter, R. 2003 Wave scattering by narrow cracks in ice sheets floating on water of finite depth. Journal of Fluid Mechanics 484 (484), 143-165.

Fox, C. \& Squire, V. A. 1990 Reflection and transmission characteristics at the edge of shore fast sea ice. Journal of Geophysical Research Oceans 95 (C7), 11629-11639.

Fox, C. \& Squire, V. A. 1994 On the oblique reflexion and transmission of ocean waves at shore fast sea ice. Philosophical Transactions of the Royal Society A347 (1682), 185-218.

Herman, A., Evers, K. U. \& Reimer, N. 2018 Floe-size distributions in laboratory ice broken by waves. The Cryosphere 12 (2), 685-699.

Kohout, A. L., Williams, M. J., Toyota, T., Lieser, J. \& Hutchings, J. 2016 In situ observations of wave-induced sea ice breakup. Deep Sea Research II 131, 22-27.

LI, Z. F., WU, G. X. \& Ji, C. Y. 2018 a Interaction of wave with a body submerged below an ice sheet with multiple arbitrarily spaced cracks. Physics of Fluids 30 (5), 057107.

LI, Z. F., Wu, G. X. \& Ji, C. Y. $2018 b$ Wave radiation and diffraction by a circular cylinder submerged below an ice sheet with a crack. Journal of Fluid Mechanics 845, 682-712.

Li, Z. F., Wu, G. X. \& SHI, Y. Y. $2018 c$ Wave diffraction by a circular crack in an ice sheet floating on water of finite depth. Physics of Fluids 30 (11), 117103.

Linton, C. M. \& Chung, H. 2003 Reflection and transmission at the ocean/sea-ice boundary. Wave Motion 38 (1), 43-52.

Linton, C. M. \& Mciver, P. 2001 Handbook of mathematical techniques for wave/structure interactions. United States: CRC Press.

Martin, P. A. \& Rizzo, F. J. 1989 On boundary integral equations for crack problems. Proceedings of the Royal Society of London A421 (1861), 341-355.

Meylan, M. H. 2002 Wave response of an ice floe of arbitrary geometry. Journal of Geophysical Research Oceans 107.

Meylan, M. H. \& Squire, V. A. 1994 The response of ice floes to ocean waves. Journal of Geophysical Research Atmospheres 99 (C1), 891-900.

Meylan, M. H. \& Squire, V. A. 1996 Response of a circular ice floe to ocean waves. Journal of Geophysical Research Oceans 101 (C4), 8869-8884.

Montiel, F., Squire, V. A. \& Bennetts, L. G. 2016 Attenuation and directional spreading of ocean wave spectra in the marginal ice zone. Journal of Fluid Mechanics 790, 492-522.

Norris, A. N. \& WANG, Z. 1994 Bending wave diffraction from strips and cracks on thin plates. Quarterly Journal of Mechanics and Applied Mathematics 47 (4), 607-627.

Peter, M. A., Meylan, M. H. \& Chung, H. A. 2004 Wave scattering by a circular elastic plate in water of finite depth: a closed form solution. International Journal of Offshore and Polar Engineering 14 (2), 81-85.

Porter, R. \& Evans, D. V. 2006 Scattering of flexural waves by multiple narrow cracks in ice sheets floating on water. Wave Motion 43 (5), 425-443.

Porter, R. \& Evans, D. V. 2007 Diffraction of flexural waves by finite straight cracks in an elastic sheet over water. Journal of Fluids and Structures 23 (2), 309-327.

Robin, G. Q. 1963 Wave propagation through fields of pack ice. Philosophical Transactions of the Royal Society A255 (255), 313-339.

Sahoo, T., Yip, T. L. \& Chwang, A. T. 2001 Scattering of surface waves by a semi-infinite floating elastic plate. Physics of Fluids 13 (11), 3215-3222.

Smith, J. A., Meylan, M. H. \& Mcphedran, R. C. 2011 Scattering by cavities of arbitrary shape in an infinite plate and associated vibration problems. Journal of Sound and Vibration 330 (16), 4029-4046. 
Squire, V. A. 2007 Of ocean waves and sea-ice revisited. Cold Regions Science and Technology 49 (2), 110-133.

Squire, V. A. 2011 Past, present and impendent hydroelastic challenges in the polar and subpolar seas. Philosophical Transactions of the Royal Society A369 (1947), 2813-2831.

Squire, V. A. 2020 Ocean wave interactions with sea ice: a reappraisal. Annual Review of Fluid Mechanics 52 (1), 37-60.

Squire, V. A. \& Dixon, T. W. 2000 An analytic model for wave propagation across a crack in an ice sheet. International Journal of Offshore and Polar Engineering 10 (3), 173-176.

Squire, V. A. \& Dixon, T. W. $2001 a$ How a region of cracked sea ice affects ice-coupled wave propagation. Annals of Glaciology 33, 327-332.

Squire, V. A. \& Dixon, T. W. $2001 b$ On modelling an iceberg embedded in shore-fast sea ice. Journal of Engineering Mathematics 40 (3), 211-226.

Squire, V. A., Dugan, J. P., Wadhams, P., Rottier, P. J. \& Liu, A. K. 1995 Of ocean waves and sea ice. Annual Review of Fluid Mechanics 27 (1), 115-168.

Squire, V. A., Robinson, W. H., Langhorne, P. J. \& Haskell, T. G. 1988 Vehicles and aircraft on floating ice. Nature 333 (6169), 159-161.

Timoshenko, S. P. \& Woinowsky, K. S. 1959 Theory of plates and shells. Singapore: McGraw-hill.

Tkacheva, L. A. 2001 Hydroelastic behavior of a floating plate in waves. Journal of Applied Mechanics and Technical Physics 42 (6), 991-996.

Tkacheva, L. A. 2004 The diffraction of surface waves by a floating elastic plate at oblique incidence. Journal of Applied Mathematics and Mechanics 68 (3), 425-436.

Wang, C. D. \& Meylan, M. H. 2004 A higher-order-coupled boundary element and finite element method for the wave forcing of a floating elastic plate. Journal of Fluids and Structures 19 (4), 557-572.

Williams, T. D. \& SQuire, V. A. 2006 Scattering of flexural-gravity waves at the boundaries between three floating sheets with applications. Journal of Fluid Mechanics 569, 113-140.

Wu, G. X. \& EATOCK TAYLOR, R. 1995 Time stepping solutions of the two-dimensional nonlinear wave radiation problem. Ocean Engineering 22 (8), 785-798. 